\title{
Unbiased Disagreement and the Efficient Market Hypothesis VERY PRELIMINARY
}

\author{
Elyes Jouini, Université Paris Dauphine \\ Clotilde Napp, CNRS and Université Paris-Dauphine
}

March 17, 2009

\begin{abstract}
Can investors with irrational beliefs be neglected as long as they are rational on average? Does unbiased diagreement lead to trades that cancel out with no consequences on prices, as implicitly assumed by the traditional models? We show in this paper that there is an important impact of unbiased disagreement on the behavior of financial markets, even though the pricing formulas are "on average" (over the states of the world) unchanged. In particular we obtain time varying, mean reverting and countercyclical (instead of constant in the standard model) market prices of risk, mean reverting and procyclical (instead of constant) risk free rates, decreasing (instead of flat) yield curves, possibly higher returns and higher risk premia in the long run (instead of a flat structure), momentum in stock returns in the short run, more variance on returns, time and state varying (instead of constant) risk sharing rules, as well as more important and procyclical trading volumes. These features seem consistent with the actual behavior of financial markets and only result from the introduction of unbiased disagreement.
\end{abstract}

\section{Introduction}

Most neoclassical asset pricing models rely on the assumption that market participants are rational and maximize their expected utility under the true probabilities of uncertain economic states. There is however a growing evidence for the 
presence of traders with biased beliefs on the markets. How does the presence of these participants affect the behavior of financial markets ?

It is widely argued that the presence of these traders can be neglected. The first main argument relies on the work of Milton Friedman (1953), and consists in saying that irrational traders need not be considered since they get eliminated in the long run. Indeed, their trading on wrong beliefs leads them to lose their wealth, they don't survive, hence cannot influence long run behavior of financial markets. The second main argument for neglecting behavioral participants relies on the fact that they should be rational on average; there is no reason for a specific systematic bias, hence investors expectations should be on average correct, their trades should cancel out and there should be no impact on financial markets. The third main argument relies on an arbitrage-like argument; the actions of the rational investors should offset the actions of the irrational ones. Prices should induce rational investors (in aggregate) to overweight (relative to market weights) the assets underweighted by the irrational investors due to their erroneous beliefs, and to underweight the assets overweighted by the irrational, thereby offsetting the price effects of the irrational investors.

There is an important body of recent literature questioning the first argument. In a general equilibrium setting, Sandroni (2000) and Blume and Easley (2006) show that with intermediate consumption irrational traders do not survive in the long run. Yan (2008a) shows that only the trader with the lowest "survival index" (a function of belief accuracy, patience and risk aversion parameters) survives in the long run. In particular, if investors have the same preferences (or if their preferences are independent from their beliefs), then those with incorrect beliefs cannot survive in the long run. However, the selection process is very slow. Kogan et al. (2006, 2008) show that survival and price impact are two different concepts. The aim of this paper is to question the second argument and to shed some light on the third argument.

The efficiency of financial markets is the principal motivation behind the interest of such questions. Indeed, if behavioral market participants impact prices and other equilibrium characterictics even when they are on average rational, then markets cannot be efficient, either informationnally or allocationnally.

We consider as our main model an equilibrium model with two groups of behavioral investors who are on average rational and we analyse to what extent these behavioral investors cancel out or on the contrary have an impact on the equilibrium characteristics. We find that this model shares some similarities with the standard rational model. In particular, in such a model, no investor gets 
eliminated in the long run (in the sense that the consumption share converges to 0 ), since no investor (or group of investors) is more wrong than the others. All the agents survive. As in the rational setting, the consumption shares of the agents remain equally distributed at all dates, which means that none of the agents wins. Finally, the market price of risk and the risk free rate are on average (over the states of the world) given by the market price of risk and the risk free rate of the rational setting.

However, the features of the setting with disagreement are very different from the features of the rational setting. The market price of risk is time and state varying. In particular, we obtain that it is countercyclical, i.e. higher in periods of recession and lower in periods of expansion. This is due to the fact that the economy is dominated by the pessimistic agent(s) in bad states of the world and by the optimistic agent(s) in good states of the world (in the sense that their relative level of absolute risk tolerance is high). There is then a "pessimistic bias" (hence a higher market price of risk) in bad states of the world and an "optimistic bias" (hence a lower market price of risk) in good states of the world. Note that this result is consistent with the observed countercyclical variations of the equity premium. Indeed, there is evidence that the equity premium is time varying and as underlined by, e.g., Campbell and Cochrane (1999) "equity risk premia seem to be higher at business cycles troughs than they are at peaks". For analogous reasons, we obtain that the risk free rate is also time and state varying. It is lower during recessions and higher during expansions. This is consistent with observed behavior since empirical studies have confirmed that the short term rate is a procyclical indicator of economic activity (see e.g. Friedman, 1986, Blanchard and Watson, 1986). The market price of risk and the risk free rate exhibit mean reversion. This is consistent with the findings of, e.g., Fama and French (1988). We also obtain that the market price of risk exhibits momentum, which is consistent with the observed positive autocorrelation in stock returns in the short run (see e.g. Jegadeesh and Titman, 1993). These conclusions are very different from the features of the rational setting in which both the risk free rate and the market price of risk are constant.

Contrarily to the standard setting, the yield curve is decreasing (Jouini et al., 2008a). More precisely, the discount rate (i.e. the rate of a zero coupon bond) is decreasing with the time horizon, and converges to the "pessimistic" discount rate, i.e. the discount rate that would prevail if the pessimistic agents had the whole endowment of the economy. This result holds even though the 
instantaneous risk free rate is at all date on average (over the states of the world) given by the rational rate. This is due to the fact that as maturity increases, the zero coupon bond, as a hedging instrument against very bad states of the world, becomes more and more desirable for the pessimistic agent, who then exerts stronger influence on its equilibrium prices.

We also obtain specific features for long term returns. In particular, for some assets, we find that the expected rolled over return (i.e. the expected return of investing one Euro in the asset and rolling it over) converges to the return that would prevail in the economy populated by the more optimistic agent only. The long term return is then higher than in the standard rational setting and higher than the instantaneous return. The long term risk premium is also higher than the rational long term risk premium and than the instantaneous risk premium. In other words, the presence of irrational traders modifies the long term relation between risk and return and introduces a distortion between the long term and the short term risk return tradeoff.

We obtain that the prices are the same as in an economy with a representative agent whose belief is a mixture of the individual ones. This implies that from the representative agent point of view the distribution of aggregate endowment is a mixture of the individual subjective distributions. The distribution of aggregate endowment then has more variance than each of the individual subjective distributions (and than the objective distribution); roughly speaking, this means that disagreement (with aggregate rationality) induces more variance than the rational setting while leaving the mean unchanged. Moreover, we obtain that the state price density is not lognormal, contrarily to the rational setting. This is consistent with the empirical literature on the state price density extracted from assets prices (see, e.g. Jackwerth and Rubinstein, 1996, or Aït-Sahalia and Lo, 1998). More precisely, we obtain that the state price density is a mixture of lognormal distributions.

We determine explicitly the efficient risk sharing rule and the trading volumes and how they differ from the standard setting. In particular, contrarily to the rational (and HARA) setting, the risk sharing rule is not linear. There is an additional time varying stochastic term, that is related to beliefs heterogeneity, each agent bearing a larger share of the risk in the states that she thinks more probable. Classical tests of efficiency that derive from the seminal work of Mace (1991) are based on the fact that any shock on the aggregate endowment should impact the agents uniformly. The Efficient Market Hypothesis would then be rejected by these tests in our setting. 
We show how beliefs heterogeneity can be assimilated with endowments heterogeneity in terms of impact on the trading volumes. In particular, if agents have the same endowments, trading is generated by beliefs divergence and the trading volumes coincide with the trading volumes that would be obtained in a standard setting with given heterogeneous endowment processes (that are not perfectly correlated with aggregate endowment). To this extent, beliefs heterogeneity generates trading. Moreover, we obtain that the trading volumes are high in very good states of the world, i.e., when aggregate endowment and prices are high. This is consistent with the empirical finding that volumes of trade are more important in bull markets.

As far as long run properties are concerned, we get that the economy essentially converges to a two scenarios economy. As already mentioned, all agents survive and, as time increases, the agents share out the states of the world, the optimistic agents consuming nearly all endowment in the "good" states of the world and the pessimistic agents consuming nearly all endowment in the "bad" states of the world. As far as the market price of risk and the risk free rate are concerned, we get that there are, in the long run, two types of states of the world: the states of the world for which the risk free rate and the market price of risk are those that would prevail in an economy made of the pessimistic agents only and the states of the world for which the risk free rate and the market price of risk are those that would prevail in an economy made of the optimistic agents only. The situation with on average rational agents is to this respect very different from the situation with e.g. one irrational agent and one rational agent $^{1}$.

We show that our model shares some similarities with a model with two possible scenarios on the mean; we highlight the common features but also the differences. In particular, we show that the two models have in common the asymptotic distributions of the state price density but have very different dynamic properties.

After analysing all these equilibrium characteristics, it seems clear that irrational agents have an important impact on the behavior of financial markets, even when they are rational on average. Disagreement matters, even when there is no bias on average.

Our main model considers logarithmic utility functions, since we know from

\footnotetext{
${ }^{1}$ Indeed, in such a setting, only the rational agent survives and the market price of risk as well as the risk free rate converge to the standard rational market price of risk and risk free rate.
} 
the literature on heterogeneous beliefs ${ }^{2}$ that this is the case for which it is most likely that on average rational beliefs have no impact on the equilibrium characteristics. In other words, if biased beliefs matter in this setting, they should matter even more with more general utility functions. This choice is also made for analytical tractability. In Section 7, we analyse the robustness of our results to other specifications of the utility functions. For general power utility functions, we show that our results remain essentially true. We also obtain additional results on the volatility, which is time and state varying and whose level can deviate from the standard rational level. On the contrary, we show that our results on the risk free rate and the market price of risk do not hold for exponential utility functions. Indeed, the properties obtained for example on the market price of risk for general power utility functions are due to the fluctuations in the (relative) levels of absolute risk tolerances (which are given by the consumption shares in the case of power or logarithmic utility functions). For CARA utility functions the relative levels of risk tolerance do not fluctuate, and we obtain in particular that the market price of risk is constant and given by the standard rational market price of risk ${ }^{3}$.

In our main model, we consider one group of optimistic agents and one group of pessimistic agents, on average rational. We analyse in the extensions if our results are robust to the presence of a third group of rational investors. Apart from the survival issues, the results remain qualitatively the same. This provides an answer to the third main argument presented above for neglecting behavioral investors. Offsetting actions by rational investors do not typically suffice to cause the price effects of wrong beliefs to disappear. This conclusion, due to Fama and French (2008), is extended here to the case of unbiased beliefs.

In our main model, beliefs biases are constant. In extension, we also consider more complex forms of disagreement, and in particular we consider the case where the investors may switch from pessimism to optimism and conversely. We show that our results pertain as long as there is some persistence in optimism and pessimism. Moreover, in general models of disagreement for which there is not necessarily a persistence in the individual biases, we show that we obtain results that are locally of the same nature.

Levy et al. (2006), Duchin and Levy (2008), Yan (2008b) are also interested in the impact of "unbiased" disagreement on prices or more generally on equilibrium characteristics and are closely related to our work. Levy et al. (2006)

\footnotetext{
${ }^{2}$ See, e.g. Rubinstein (1975) or Jouini and Napp (2007).

${ }^{3}$ This case is essentially the continuous time analog of Levy et al. (2006).
} 
show that if investors have heterogeneous but unbiased beliefs about the expected returns, the homogeneous CAPM pricing holds. Duchin and Levy (2008) analyse the impact of disagreement on the return variances in a mean-variance setting. More precisely, the paper shows that contrarily to the disagreement on the mean, unbiased disagreement on the variance has systematic pricing effects. Yan (2008b) analyses the impact of independent biases in investors demand functions on assets prices. He first shows that independent biases affect prices if investor's demand function is a non linear function of the bias (as in the case studied by Duchin and Levy, 2008). Then he shows in a two period setting that even if the demand function is linear in the bias, the fluctuation of the wealth distribution leads to stock return negative autocorrelation.

Related papers also include Abel (1989), Cabrales and Hoshi (1996), Calvet et al. (2002), Detemple and Murthy (1994), Zapatero (1998), Berrada (2006), Jouini and Napp (2006, 2007), and Gollier (2007), that deal with the equilibrium characteristics in an heterogeneous beliefs framework. Our paper is to be contrasted to the works of Scheinkman and Xiong (2003), Dumas et al. (2009), Li (2007), David (2008) who consider specific models of beliefs divergence and updating, while our aim is to explore the impact of "noisy" beliefs, independently of a specific dynamics for beliefs formation. Our paper is also to be contrasted to the strand of disagreement literature in which investors learn from prices like in e.g., Admati (1985), Biais et al. (2003) or DeMarzo and Skiadas (1998).

All proofs are in the Appendices (Appendix A for the main model, Appendix $\mathrm{B}$ for the extensions).

\section{The model}

We want to analyse to what extent behavioral agents who are on average rational can be neglected or on the contrary have an impact on the equilibrium characteristics. We adopt on purpose a (main) model that is as simple as possible, and consider more complex settings and extensions in Section 7. Our utility functions are logarithmic. The setting is dynamic since we want to analyse the dynamic properties of the equilibrium. The horizon is infinite since we want to consider survival properties or more generally long run properties. As far as disagreement is concerned, our model can be seen as the dynamic analog of the two agents problem with lognormally distributed aggregate endowment and disagreement about its average level.

More precisely, we consider a continuous-time pure exchange Arrow-Debreu 
economy, with a single consumption good and two risk averse agents (or groups of agents) trying to maximize their expected utility from future consumption. We assume that both agents have the same utility function for consumption, the same time preference rate but that they can differ in their subjective beliefs about the future of the economy. More precisely, a filtered probability space $\left(\Omega, F,\left(F_{t}\right), P\right)$ describing uncertainty is given and each agent has a von Neumann Morgenstern utility for future consumption of the form

$$
U_{i}(c)=E^{Q_{i}}\left[\int_{0}^{\infty} \exp (-\rho t) u\left(c_{t}\right) d t\right]
$$

where $\rho$ represents the time preference rate parameter, $u$ is the common utility function and the subjective belief of each agent is represented by the subjective probability measure $Q_{i}$, equivalent to the initial probability $P$. We let $M^{i}$ denote the density of $Q_{i}$ with respect to $P$, i.e., $\frac{d Q_{i}}{d P}=M^{i}$, so that the utility of agent $i$ for the consumption stream $c$ can be written in the form $U_{i}(c)=E\left[\int_{0}^{\infty} M_{t}^{i} \exp (-\rho t) u\left(c_{t}\right) d t\right]$.

We let $e^{*^{i}}$ denote the endowment process of agent $i$ and we let $e^{*} \equiv \sum_{i=1}^{2} e^{*^{i}}$ denote the aggregate endowment process. We make the assumption that the processes $e^{*}$ and $M^{i}$ satisfy the following stochastic differential equations

$$
\left\{\begin{array}{c}
d e_{t}^{*}=\mu e_{t}^{*} d t+\sigma e_{t}^{*} d W_{t} \\
d M_{t}^{i}=\delta_{i} M_{t}^{i} d W_{t}
\end{array}\right.
$$

where $W$ is a standard unidimensional $\left(\left(F_{t}\right), P\right)$-Brownian motion and $\left(\mu, \sigma,\left(\delta_{i}\right)\right) \in$ $\left(\mathbb{R}_{+}\right)^{2} \times \mathbb{R}^{2}$ are given constants. The assumption on $e^{*}$ means that $e^{*}$ is a geometric Brownian motion with drift. In such a context, Agent 1 and Agent 2 both know that the volatility parameter is given by $\sigma$ but have different beliefs about the constant growth rate $\mu$. More precisely, by Girsanov Theorem, we have

$$
\left\{\begin{aligned}
d e_{t}^{*} & =\left(\mu+\sigma \delta_{1}\right) e_{t}^{*} d t+\sigma e_{t}^{*} d W_{t}^{1} \\
d e_{t}^{*} & =\left(\mu+\sigma \delta_{2}\right) e_{t}^{*} d t+\sigma e_{t}^{*} d W_{t}^{2}
\end{aligned}\right.
$$

where for $i=1,2, W_{t}^{i} \equiv W_{t}-\delta_{i} t$ is a Brownian motion under $Q_{i}$, which means that Agent 1 believes that the aggregate endowment growth rate is given by $\mu_{1} \equiv \mu+\sigma \delta_{1}$, whereas Agent 2 believes it is given by $\mu_{2} \equiv \mu+\sigma \delta_{2}$ and both agents agree on the volatility parameter $\sigma$. The parameter $\delta_{i}=\frac{\mu_{i}-\mu}{\sigma}$ then measures 
investor $i$ 's error in his perceived economic growth (normalized by the level of risk $)^{4}$.

Since for Agent $i$, the instantaneous expected growth rate is given by $\mu+\sigma \delta_{i}$, we shall refer to rationality when $\delta_{i}=0$, and to optimism (resp. pessimism) when $\delta_{i}>0$ (resp. $\delta_{i}<0$ ). We assume that there is no aggregate bias, i.e., the agents are rational on average with $\delta_{1}+\delta_{2}=0$. In this case, one agent is optimistic $\left(\delta_{1} \equiv \delta>0\right)$, the other agent is pessimistic $\left(\delta_{2}=-\delta\right)$, and the agents have the same "level of irrationality" $\left(\left|\delta_{1}\right|=\left|\delta_{2}\right|\right)$. For the sake of comparison, we shall sometimes mention the case with a bias, $\delta_{1}+\delta_{2} \neq 0$, an optimistic bias when $\delta_{1}+\delta_{2}>0$, a pessimistic bias when $\delta_{1}+\delta_{2}<0$.

Note that in our main model, the biases $\delta_{i}$ are constant. This choice is first made for simplicity. Indeed, the most simple and natural extension of a model where the true growth rate is a constant $\mu$ to the case with disagreement (with on average rational beliefs) is the setting with two agents, one agent believing that the growth rate is $\mu+\sigma \delta$ and the other believing that it is $\mu-\sigma \delta$. The restriction implied by such a modelling is the fact that one group of agents systematically overestimates the growth rate while the other group of agents systematically underestimates it. This restriction is consistent with the interpretation of the bias on the beliefs as a behavioral bias characterising the behavior of the individual towards risk, like the individual distorsions of the underlying probability distributions, introduced in the recent literature of decision theory. With such an interpretation, an individual is more or less pessimistic in the same way as she is more or less risk tolerant, or impatient. If the bias corresponds to a behavioral bias having decision theoretical foundations, then it is consistent to suppose that the bias is persistent, one group of agents remaining optimistic and the other group of agents remaining pessimistic. Our notion of optimism/pessimism coincides in our setting with the notions of optimism/pessimism adopted by e.g., Yaari (1987), Chateauneuf and Cohen (1994), Dieciedue and Wakker (2001)5.

\footnotetext{
${ }^{4}$ The fact that both agents agree on the volatility parameter is implied by the assumption that the individual probabilities $Q_{i}$ are equivalent to the initial one $P$. This assumption is quite natural. Note that if the $Q_{i}$ were absolutely continuous with respect to $P$ and not equivalent, and if there existed an event $A$ with a positive probability for Agent 1 and a zero probability for Agent 2, equilibrium could not be reached since the demand of Agent 1 would be infinite in event A. Moreover, as already noticed by Basak (2000) or Yan (2008a), this parametrization is consistent with the insight from Merton (1980) that the expected return is harder to estimate than the variance.

${ }^{5}$ Chateauneuf and Cohen (1994) relate it to the notion of First Stochastic Dominance, while Yaari (1987) and Diecidue and Wakker (2001) relate it to the notion of Monotone Likelihood
} 
Note that constant parameters ${ }^{6} \delta_{i}$ are also adopted in, e.g., Kogan et al. (2006) or Yan (2008a). However, we analyse the robustness of our results to more general models of unbiased disagreement in Section 7.

We assume that individual endowments are perfectly correlated with aggregate endowment, i.e. of the scalar form $e^{*^{i}}=w_{i} e^{*}$ with $w_{1}+w_{2}=1$. We consider logarithmic utility functions $u$ in the main model and we will analyse in Section 7 the robustness to other utility functions in the HARA class. We will also consider as an extension a model where in addition to the pessimistic group and the optimistic group, there is also a rational group.

An Arrow-Debreu equilibrium relative to the beliefs $M^{i}$ is defined by a positive density price process $q^{*}$ and a pair of optimal consumption plans $\left(y^{*^{i}}\right)_{i=1,2}$ such that markets clear, i.e.,

$$
\left\{\begin{array}{c}
y^{*^{i}}=y_{i}\left(q^{*}, M^{i}, e^{*^{i}}\right) \\
\sum_{i=1}^{2} y^{*^{i}}=e^{*}
\end{array}\right.
$$

where $y_{i}(q, M, e) \equiv \arg \max _{E\left[\int_{0}^{T} q_{t}\left(c_{t}-e_{t}\right) d t\right] \leq 0} E\left[\int_{0}^{T} M_{t} \exp (-\rho t) u\left(c_{t}\right) d t\right]$.

In such a setting, it is easy to obtain (see e.g. Jouini and Napp, 2007, Detemple and Murthy, 1994) that there exists a unique equilibrium given by

$$
\begin{aligned}
& y_{1}^{*}=\left(\frac{w_{1} M^{1}}{w_{1} M^{1}+w_{2} M^{2}}\right) e^{*}, \quad y_{2}^{*}=\left(\frac{w_{2} M^{2}}{w_{1} M^{1}+w_{2} M^{2}}\right) e^{*}, \\
& q^{*}=\left(w_{1} M^{1}+w_{2} M^{2}\right) \exp (-\rho t)\left(\frac{1}{e^{*}}\right) .
\end{aligned}
$$

We shall refer to $M \equiv\left(w_{1} M^{1}+w_{2} M^{2}\right)$ as the consensus belief density. We consider in the main model the case of equal initial endowment, i.e. $w_{1}=w_{2}$.

\section{Consumption shares, wealth shares and survival issues}

For ease of exposition, we let $\tau_{i} \equiv \frac{y^{*^{i}}}{e^{*}}$ denote the consumption share of agent $i$. Note that $\tau_{i}$ also corresponds to the individual relative level of absolute risk

Ratio. These notions coincide in our setting.

${ }^{6}$ The choice of constant parameters can also model "tastes for assets" as in e.g. Fama and French (2008). In this case, a positive $\delta$ would correspond to the agents who like the asset and a negative $\delta$ to the agents are those who dislike the asset. 
tolerance given by $-\frac{u^{\prime}\left(y^{*^{i}}\right)}{u^{\prime \prime}\left(y^{*^{i}}\right)}\left[\sum_{i=1}^{2}-\frac{u^{\prime}\left(y^{*^{i}}\right)}{u^{\prime \prime}\left(y^{*^{i}}\right)}\right]^{-1}$.

In the standard rational case, the consumption share of each agent is time and state independent and equal to $1 / 2$. In the case with one rational and one irrational agent, we know (see e.g. Kogan et al., 2006) that the irrational agent becomes extinct in the sense that her consumption share converges to 0 and that the consumption share of the rational agent converges to 1 (almost surely). More generally, if there is a bias on average, one agent being more wrong than the other, the more rational agent "wins" in the very long run: when $\left|\delta_{1}\right|>\left|\delta_{2}\right|$ then $\tau_{1}(t)$ converges to 0 and $\tau_{2}(t)$ converges to 1 almost surely (see, e.g., Yan, 2008a). The economy ends up being dominated by the more rational agent. As expected, the situation with no bias on average is very different.

\section{Proposition 1. Distribution of Consumption Shares}

1. At all date $t$, we have $E\left[\tau_{i}(t)\right]=1 / 2$.

2. For all date $t$, the random variables $\tau_{1}(t)$ and $\tau_{2}(t)$ have the same distribution. It is given by the following density on $[0,1]$

$$
f_{\tau_{i}(t)}(x)=\frac{1}{2 \sqrt{2 \pi t} \delta} \frac{1}{x(1-x)} \exp \left(-\frac{1}{2} \frac{\left[\log \left(\frac{x}{1-x}\right)\right]^{2}}{4 \delta^{2} t}\right) \mathbf{1}_{[0,1]}(x) .
$$

This distribution is symmetric with respect to $1 / 2$.

3. For all $\varepsilon>0, P\left(\tau_{i}(t) \geq 1-\varepsilon\right)$ as well as $P\left(\tau_{i}(t) \leq \varepsilon\right)$ increase towards $1 / 2$ as $t$ increases.

Proposition 1 implies in particular that the consumption shares of both agents are on average (over the states of the world) given by the standard rational consumption shares (Point 1.). Moreover, none of the agents "wins". At all dates, consumption is equally shared, i.e., the consumption shares of the agents are identically distributed. Figure 1 illustrates the situation at different dates. Point 3. implies that in the long run, both agents survive. This is due to the fact that no agent is more wrong than the other ${ }^{7}$. More precisely, there is, in the long run, a probability near 1/2 that Agent 1 dominates the economy and a probability near $1 / 2$ that Agent 2 dominates the economy. This implies

\footnotetext{
${ }^{7}$ In Yan (2008)'s terminology, the agents have the same survival index.
} 
that in the long run the economy is dominated by one of the two agents with a probability near 1 .

However, these properties of "equal sharing" of consumption among agents at all dates pertain in distribution only, as shown in the next proposition.

Proposition 2. 1. The optimal consumption shares are time varying and stochastic. They are given by

$$
\tau_{1}(t)=\frac{1}{2} \frac{M_{t}^{1}}{M_{t}}=\frac{\exp \left(2 \delta W_{t}\right)}{1+\exp \left(2 \delta W_{t}\right)}, \tau_{2}(t)=\frac{1}{2} \frac{M_{t}^{2}}{M_{t}}=\frac{1}{1+\exp \left(2 \delta W_{t}\right)} .
$$

2. Each agent has a larger share of aggregate endowment in the states that she thinks more probable. Indeed, $\tau_{1}(t)>\tau_{2}(t)$ in the states of the world for which $M_{t}^{1}>M_{t}^{2}$.

3. There is an optimistic bias in the good states of the world and a pessimistic bias in the bad states of the world. More precisely, we have $\tau_{1}>\tau_{2}$ if and only if $\log e_{t}^{*}>E\left[\log e_{t}^{*}\right]$. Moreover, there is a shift in favor of (resp. against) optimistic agents following good (resp. bad) news.

4. The economy is dominated by the optimistic agents for very high levels of aggregate endowment and dominated by the pessimistic agents for very low levels of aggregate endowment. More precisely, the share $\tau_{1}$ is near zero when $e^{*}$ is small, and it is near one when $e^{*}$ takes very high positive values.

5. The stochastic processes $\tau_{1}(t)$ and $\tau_{2}(t)$ exhibit mean-reversion. More precisely, they satisfy the following Stochastic Differential Equations

$$
\begin{aligned}
& d \tau_{1}(t)=2 \delta \tau_{1}\left(1-\tau_{1}\right)\left[\delta\left(1-2 \tau_{1}\right) d t+d W_{t}\right] \\
& d \tau_{2}(t)=-2 \delta \tau_{2}\left(1-\tau_{2}\right)\left[\delta\left(2 \tau_{2}-1\right) d t+d W_{t}\right] .
\end{aligned}
$$

Note first that individual consumption shares are directly related to individual biases in beliefs. The consumption shares are nomore constant as in the standard setting but time-varying and stochastic. We retrieve, as in the previous proposition, that at all date, the probability that Agent 1 has a larger share of aggregate consumption than Agent 2 is equal to the probability that Agent 2 has a larger share of aggregate consumption. However, we obtain that at the equilibrium, each agent has a larger share of aggregate consumption in the states that 
she thinks more probable, which is intuitive. These are the good states of the world for the optimistic agent and the bad states of the world for the pessimistic agent. In fact, the consumption share of the optimistic (resp. pessimistic) agent is an increasing (resp. decreasing) function of aggregate endowment, approaching 0 and 1 (resp. 1 and 0 ) as the level of aggregate endowment shifts to 0 and infinity respectively. This implies that the consumption shares are biased in favor of the optimistic agents in the good states of the world, and in favor of the pessimistic agents in the bad states of the world. Moreover, for very good (resp. very bad) states of the world, the optimistic agents (resp. pessimistic) dominate the economy, i.e. their consumption share is near one. Note that the consumption share of the optimistic (resp. pessimistic) agent is also an increasing (resp. decreasing) function of $W_{t}$, which leads to the results on the impact of positive or negative shocks in Point 3. Point 5. also illustrates the impact of shocks on the consumption shares. The main content of Point 5 . is the mean reversion property. Consumption shares have a tendency to revert to their average level of $1 / 2$. However, as seen in Proposition 1 , each consumption share either converges to 0 or 1 asymptotically along each trajectory.

Note that the individual consumption shares $\tau_{i}$ also correspond to the individual wealth shares of agent $i$, given by $E\left[\int_{t}^{+\infty} q_{s}^{*} y_{s}^{*^{i}} d s\right]\left[E\left[\int_{t}^{+\infty} q_{s}^{*} e_{s}^{*} d s\right]\right]^{-1}$, so that all the results obtained on the instantaneous consumption shares also hold for the wealth shares.

The fact that the consumption shares or the wealth shares (which represent the relative levels of risk tolerance) fluctuate in time and in state of the world has an impact on asset pricing, that we analyze in the next section.

\section{Asset pricing}

In order to deal with asset pricing issues, we suppose that agents can continuously trade in a riskless asset and in risky stocks. We let $S^{0}$ denote the riskless asset price process with dynamics $d S_{t}^{0}=r_{t}^{f} S_{t}^{0} d t$. We consider a risky asset $S$ with given volatility level ${ }^{8} \sigma_{s}$, and with dynamics ${ }^{9} d S_{t}=S_{t}\left[\mu_{s} d t+\sigma_{s} d W_{t}\right]$. The risk free rate process $r^{f}$, as well as the stock return drift $\mu_{s}$ are to be determined

\footnotetext{
${ }^{8}$ Note that by market completeness such an asset with a given volatility level always exists.

${ }^{9}$ Note that in the logarithmic setting, the price of the stock whose dividends are the aggregate endowment, is given by $S_{t}=\frac{1}{\rho} e_{t}^{*}$, hence there is no impact of unbiased disagreement on the price of this stock. This is not true in the general power utility setting (see Section7).
} 
endogenously in equilibrium. As in the standard setting we obtain that the risk free rate $r^{f}$ and the Market Price of Risk $\left(M P R \equiv \frac{\mu_{s}-r^{f}}{\sigma_{s}}\right)$ are directly related to the expression of the equilibrium price $q_{t}^{*} \equiv \exp (-\rho t) \frac{M_{t}}{e_{t}^{*}}$ since $r_{f}=-\mu_{q^{*}}$ and $M P R=-\sigma_{q^{*}}$. In the standard rational setting, the risk free rate and the market price of risk are time and state independent and given by $r^{f}$ (stdd) $=$ $\rho+\mu-\sigma^{2}$ and $M P R$ (stdd) $=\sigma$. In Jouini and Napp (2007), expressions for the risk free rate and the market price of risk are obtained in a very general setting with heterogeneous beliefs. In the next proposition, we analyse their properties in our specific setting.

Proposition 1. Risk free rate and market price of risk

1. The risk free rate and the market price of risk are time and state varying. More precisely, we have, for all $t$

$$
\begin{aligned}
r_{t}^{f} & =r^{f}(s t d d)+\sigma\left(\tau_{1}(t)-\tau_{2}(t)\right) \delta \\
M P R_{t} & =M P R(s t d d)-\left(\tau_{1}(t)-\tau_{2}(t)\right) \delta
\end{aligned}
$$

where $r^{f}($ stdd $)=\rho+\mu-\sigma^{2}$ and $M P R($ stdd $)=\sigma$.

2. The distribution of $M P R_{t}$ (resp. of $r_{t}^{f}$ ) is symmetric with respect to $M P R($ stdd $)\left(\right.$ resp. $r^{f}\left(\right.$ stdd). In particular, $E\left[r_{t}^{f}\right]=r_{t}^{f}($ stdd $)$ and $E\left[M P R_{t}\right]=$ $M P R_{t}($ stdd) .

3. The market price of risk is countercyclical: it is lower in states of the world where $\tau_{1}(t)>\tau_{2}(t)$, i.e. in "good" states of the world and higher in states of the world where $\tau_{2}(t)>\tau_{1}(t)$, i.e. in "bad"states of the world. Moreover, "good news" (resp. "bad news") decrease (resp. increase) the market price a risk.

4. The risk free rate is procyclical: it is higher in states of the world where $\tau_{1}(t)>\tau_{2}(t)$, i.e. in "good" states of the world and lower in states of the world where $\tau_{2}(t)>\tau_{1}(t)$, i.e. in "bad" states of the world. Moreover, "good news" (resp. "bad news") increase (resp. decrease) the risk free rate.

5. The market price of risk exhibits momentum. 
6. The risk free rate and market price of risk stochastic processes exhibit mean reversion. More precisely, they satisfy the following Stochastic Differential Equations

$$
\begin{aligned}
d M P R_{t} & =-4 \delta^{2} \tau_{1}(t) \tau_{2}(t)\left[\left(M P R_{t}-E\left[M P R_{t}\right]\right) d t+d W_{t}\right] \\
d r_{t}^{f} & =-4 \delta^{2} \tau_{1}(t) \tau_{2}(t)\left[\left(r_{t}^{f}-E\left[r_{t}^{f}\right]\right) d t-\sigma d W_{t}\right]
\end{aligned}
$$

The distributions of the market price of risk and of the risk free rate are symmetric with respect to the standard quantities, and we retrieve on average over the states of the world the standard market price of risk and the standard risk free rate, which is consistent with the fact that our agents are on average rational. Figure 1 illustrates this result. However, the behavior of the risk free rate and of the market price of risk is inheritted from the behavior of the consumption shares (or the risk tolerances). Letting $r_{t}^{i, f}$ and $M P R^{i}$ denote the risk free rate and the market price of risk that would prevail if Agent $i$ had all the endowment, we easily get that $r_{t}^{1, f}=r_{t}^{f}$ (stdd) $+\sigma \delta, r_{t}^{2, f}=r_{t}^{f}$ (stdd) $-\sigma \delta$, $M P R^{1}=M P R(s t d d)-\delta$ and $M P R^{2}=M P R(s t d d)+\delta$. Point 1 . then shows that the risk free rate and the market price of risk in our setting are consumption shares weighted averages of the individual quantities (see also Jouini and Napp, 2007 or Detemple and Murthy, 1994). In particular, the risk free rate and the market price of risk lie inside the range bounded by the two limiting cases ${ }^{10}$, i.e. $r^{f} \in\left[r^{2, f}, r^{1, f}\right]$ and $M P R \in\left[M P R^{1}, M P R^{2}\right]$. Since we know that the consumption shares are biased in favor of the optimistic agents (resp. pessimistic agents) in good states of the world (resp. bad states of the world), we obtain that the risk free rate and the market price of risk are biased in favor of the risk free rate and the market price of risk of the optimistic (resp. pessimistic) agents in good states of the world (resp. bad states of the world). In other words there is an optimistic bias in good states of the world and a pessimistic bias in bad states of the world. Moreover, we have seen in Proposition 2 that positive (resp. negative) shocks lead to an increase of the weight of the optimistic (resp. pessimistic) agents. This leads to the results of Points 3. and 4. The market price of risk is countercyclical. This result is consistent with the observed variations of the equity premium. Indeed, there is evidence that the equity premium is time varying and as underlined by, e.g., Campbell and Cochrane (1999) "equity

\footnotetext{
${ }^{10}$ Note that in the case of power utility functions, the result on the market price of risk remains valid, i.e., $M P R \in\left[M P R^{1}, M P R^{2}\right]$ but the risk free rate can lie outside the interval $\left[r^{2, f}, r^{1, f}\right]$. See Section 7 for more details.
} 
risk premia seem to be higher at business cycles troughs than they are at peaks". For analogous reasons, we obtain that the risk free rate is also time and state varying. It is lower during recessions and higher during expansions. This is also consistent with observed behavior since empirical studies have confirmed that the short term rate is a procyclical indicator of economic activity (see e.g. Friedman, 1986, Blanchard and Watson, 1986). Point 5. indicates that there is momentum, i.e., a tendency for high returns (resp. low returns) to continue to be high (resp. low) over a short period of time. This means that we obtain short run positive autocorrelation ${ }^{11}$. This is due to the fact that in our setting, high (resp. low) observed returns are (on average) associated to a pessimistic (resp.optimistic) bias; a pessimistic (resp. optimistic) economy remains pessimistic in the short run which tends to continue to generate (on average) high future returns in the short run. This is consistent ${ }^{12}$ with empirical evidence on momentum (see e.g. Jegadeesh and Titman, 1993). These conclusions are very different from the features of the rational setting in which both the risk free rate and the market price of risk are constant. These results are illustrated in Figure 2 .

Moreover, we obtain in Point 6 . that the market price of risk and the risk free rate exhibit mean reversion. Note that according to Proposition 1, we easily get that there are two possible asymptotic scenarios ${ }^{13}$ for the risk free rate and the market price of risk, i.e. as time $t$ increases, the probability to have $r_{t}^{f}=r_{t}^{1, f}$ as well as $M P R=M P R^{1}$ increases towards $1 / 2$. The same applies to the probability to have $r_{t}^{f}=r_{t}^{2, f}$ as well as $M P R=M P R^{2}$.

We now analyse the behavior of the yield curve. In the standard setting, the yield curve is flat; for all date $t$, the rate of return associated to a zero coupon bond maturing at date $t$ is given by $R$ (stdd) $=\rho+\mu-\sigma^{2}$. The discount rate is the same as the instantaneous risk free rate. If the pessimistic (resp. the optimistic) agents have all the endowment, then the discount rate is given by $R_{\text {pess. }}$ (stdd) $=\rho+\mu-\sigma^{2}-\delta \sigma$ (resp. $R_{\text {opt. }}($ stdd $\left.)=\rho+\mu-\sigma^{2}+\delta \sigma\right)$. Jouini et al. (2008a) analyses the yield curve in a general setting with heterogeneous beliefs. Adapting the results therein to our specific setting leads to the following proposition.

\footnotetext{
${ }^{11}$ Note that Yan (2008b) obtains negative autocorrelation on stocks prices, whereas we obtain positive short run autocorrelation. This is mainly due to the fact that Yan (2008b)'s model is a two period model.

${ }^{12}$ There is a literature relating disagreement and momentum. It usually relies on gradual information flow (or limited attention or underreaction). See e.g. Hong and Stein (2007).

${ }^{13} \mathrm{We}$ analyse in detail the analogy with an economy with scenarios in Section 7.
} 
Proposition 2. Discount rates (Jouini et al., 2008a)

The discount rate $R_{t} \equiv-\frac{1}{t} \log E\left[q_{t}^{*}\right]$ is given for all $t$ by

$$
R_{t}=\rho+\mu-\sigma^{2}-\frac{1}{t} \log \left[\frac{1}{2} \exp (-\delta \sigma t)+\frac{1}{2} \exp (+\delta \sigma t)\right] .
$$

In particular, the yield curve $\left(R_{t}\right)_{t}$ is decreasing with $t$ and we have

$$
R_{0}=r^{f}(\text { stdd })=\rho+\mu-\sigma^{2}, \quad R_{\infty}=R_{\text {pess. }}(\text { stdd })=\rho+\mu-\sigma^{2}-\delta \sigma .
$$

This means that the yield curve is not flat as in the standard setting, but decreasing. The long run discount rate is always the pessimistic rate, i.e., the constant rate that would prevail if Agent 1 had all the endowment. The (very) short run discount rate $R_{0}$ is the rational rate. We have seen in Proposition 1 that the risk free rate is on average given by the rational rate since for all $t$, $E\left[r_{t}^{f}\right]=\rho+\mu-\sigma^{2}$. We have also seen that none of the agents vanishes, that for all $t$, the consumption shares of Agent 1 and Agent 2 at time $t$ are identically distributed and that the risk free rate either converges to the risk free rate of the pessimistic agent or to the risk free rate of the optimistic agent (with the same probability). However, the discount rate converges to the discount rate of the more pessimistic agent. This apparent contradiction is due to the fact that the behavior of the instantaneous risk free rate $r_{t}^{f}$ is given by the consumption shares at time $t$. As already seen, since no agent wins, the risk free rate is on average given by the rational rate. On the contrary, as time $t$ increases, the discount rate $R_{t}$ between time 0 and time $t$ gets closer to the discount rate of the pessimistic agent, since he is the agent who values the most a zero coupon bond of maturity $t$ : indeed, a zero coupon bond is a good hedging instrument against the bad states of the world and these bad states of the world become more and more likely as $t$ increases for the pessimistic agent.

We now turn to considerations about the long term returns of the risky assets. In the standard setting, we know that the instantaneous return for an asset $S$, whose volatility level is given by $\sigma_{S}$, is constant and given by $\mu_{s t d d}=r_{s t d d}^{f}+\sigma_{S} \sigma$, for all $t$, hence the cumulative return is given by $C R_{T}^{S}(s t d d) \equiv \frac{1}{T} \log E\left[S_{T}\right]=$ $\frac{1}{T} \log E\left[\exp \left\{\left(r_{\text {stdd }}^{f}+\sigma_{S} \sigma-\frac{\sigma_{S}^{2}}{2}\right) T+\sigma_{S} W_{T}\right\}\right]=r_{\text {stdd }}^{f}+\sigma_{S} \sigma$. It is constant and given by the instantaneous return $\mu_{\text {stdd }}=\rho+\mu-\sigma^{2}+\sigma \sigma_{S}$. In our setting, we easily obtain that the instantaneous return at time $t$ is the consumption share weighted average of the instantaneous rates that would prevail if agent $i$ had all 
the endowment, and given by $\mu_{S}(t)=\rho+\mu-\sigma^{2}+\sigma \sigma_{S}+\left(\tau_{1}-\tau_{2}\right) \delta\left(\sigma-\sigma_{S}\right)$. The next proposition shows that the long run cumulative return in the setting with disagreement and in the standard setting can be different.

Proposition 3. For $0 \leq \sigma_{S} \leq \sigma$, the long term cumulative return is given by $C R_{\infty}^{S} \equiv \lim _{T} C R_{T}^{S}=\mu_{\text {stdd }}+\delta\left(\sigma-\sigma_{S}\right)$ with $\mu_{\text {stdd }}=\rho+\mu-\sigma^{2}+\sigma \sigma_{S}$. It is the cumulative return that would prevail with only the optimistic agent present in the economy. In particular, $C R_{\infty}^{S} \geq C R^{S}\left(\right.$ stdd) and $C R_{\infty}^{S} \geq \mu_{S}(t)$.

This means that in the setting of the proposition, the long term return is higher than the (instantaneous or long term) return in the standard setting. It is also higher than the instantaneous return. For such an asset, consider now the risk premium between time 0 and $T$, that we define by $r p_{T}^{S} \equiv \frac{1}{T} \log E\left[S_{T}\right]-R_{T}$. In the standard setting, it is given by $r p_{T}^{S}(s t d d)=\sigma \sigma_{S}$, and coincides, for all $T$, with the instantaneous risk premium. The results in our setting can be different.

Corollary 4. For $0 \leq \sigma_{S} \leq \sigma$, we have $r p_{\infty}^{S} \equiv \lim _{T} r p_{T}^{S}=r p(s t d d)+\delta\left(\sigma-\sigma_{S}\right)+$ $\delta \sigma$, where $\operatorname{rp}($ stdd $)=\sigma \sigma_{S}$.

This means that in the setting of the proposition, the relation between risk and return is modified in the very long run. The (very) long term risk premium is higher than the standard long term risk premium. It is also higher than the instaneous risk premium, which, in our setting, is given by $\sigma \sigma_{S}-\left(\tau_{1}(t)-\tau_{2}(t)\right) \delta \sigma_{S}$. A possible interpretation is that there is in the long term an additional risk, a sentiment risk due to disagreement, that modifies the standard risk return relation.

\section{Consensus belief and state price density}

We have seen through Equations (2.1) and (2.2) that the equilibrium price process $q^{*}$ (or the state price density) in our economy is the same as in an equivalent homogeneous economy with aggregate endowment $e^{*}$ and with a consensus belief $M$. To describe this consensus belief and analyse its properties appears then as particularly important.

The consensus belief can be represented by the probability measure $Q$, with

density $\frac{d Q}{d P} \equiv M$, and for the consensus agent, aggregate endowment follows the dynamics

$$
d e_{t}^{*}=\left(\mu+\tau_{1} \sigma \delta-\tau_{2} \sigma \delta\right) e_{t}^{*} d t+\sigma e_{t}^{*} d W_{t}^{Q}
$$


where $W^{Q}$ is a Brownian Motion for the consensus agent. Note that for the consensus agent, the instantaneous expected growth rate is time varying and stochastic since it is given by $\left(\mu+\tau_{1}(t, \omega) \sigma \delta-\tau_{2}(t, \omega) \sigma \delta\right)$. The drift evolves then smoothly between the two bounds $\mu_{1}$ and $\mu_{2}$. From the consensus agent point of view, this "regime shifting model" can be seen as a smooth version of the regime switching model of e.g., David and Veronesi (2002). Moreover, according to Proposition 2, Equation (5.1) implies that the consensus agent is optimistic in good states of the world and pessimistic in bad states of the world. There is no bias at the aggregate level in the sense that on average the consensus agent is neither optimistic nor pessimistic since $E\left[\left(\mu+\tau_{1} \sigma \delta-\tau_{2} \sigma \delta\right)\right]=\mu$. Note that according to Proposition 2, the instantaneous expected growth rate of the consensus agent exhibits mean reversion.

In the standard rational setting, the random variable $\log e^{*}$ is Gaussian with mean $\left(\mu-\frac{\sigma^{2}}{2}\right) t$ and variance $\sigma^{2} t$. In our setting, for Agent 1 , the random variable $\log e_{t}^{*}$ is Gaussian with mean $\left(\mu+\sigma \delta-\frac{\sigma^{2}}{2}\right) t$ and variance $\sigma^{2} t$, and for Agent 2 , it is also Gaussian with the same variance and mean $\left(\mu-\sigma \delta-\frac{\sigma^{2}}{2}\right) t$. Since $M=\frac{1}{2}\left(M^{1}+M^{2}\right)$, we easily obtain that the distribution of $\log e_{t}^{*}$ for the consensus agent is a mixture of the individual subjective distributions, with equal weights ${ }^{14}$. Since a mixture of Gaussian random variables is not Gaussian, the distribution of $\log e^{*}$ for the consensus agent is not Gaussian; in particular, if the divergence $\delta$ is large enough, the distribution of $\log e^{*}$ for the consensus agent is bimodal. We easily get that $E^{Q}\left[\log e_{t}^{*}\right]=t \mu$, and $\operatorname{Var}^{Q}\left[\log e_{t}^{*}\right]=\sigma^{2} t+t^{2} \sigma^{2} \delta^{2}$. This means in particular that the distribution of $\log e_{t}^{*}$ has the same mean and more variance than the objective distribution. It also has more variance than each of the individual subjective distributions. Roughly speaking, this means that on average rational behavioral beliefs induce more variance while leaving the mean unchanged.

As far as the state price density $q^{*}$ is concerned, we know that in the standard rational setting, the state price density, given by $q^{*}=\exp (-\rho t) u^{\prime}\left(e^{*}\right)$, is lognormal with $\log q^{*} \sim \mathcal{N}\left(\left(-\rho-\mu+\frac{\sigma^{2}}{2}\right) t, \sigma^{2} t\right)$. In our setting, the state price density $q^{*}=\exp (-\rho t) M u^{\prime}\left(e^{*}\right)$ is a weighted average of the state price densities that would prevail if one of the agents had all the endowment, i.e. $q^{*}=\frac{1}{2} q^{*^{1}}+\frac{1}{2} q^{*^{2}}$ where $q^{*^{i}}=\exp (-\rho t) M^{i} u^{\prime}\left(e^{*}\right)$. Note that each state price

\footnotetext{
${ }^{14}$ The behavioral properties of the representative agent in a setting with heterogeneous beliefs are analysed in Jouini and Napp (2008b).
} 
density $q^{*^{i}}$ is lognormally distributed. The state price density in our setting is a mixture of two lognormal distributions. In particular, it is not lognormal as in the standard setting, which is consistent with the empirical literature on the state price density extracted from assets prices (see, e.g. Jackwerth and Rubinstein, 1996, or Aït-Sahalia and Lo, 1998).

\section{Efficient risk sharing rules and trading volumes}

At the individual level, we obtain through Equations (2.1) and (2.2) that there is a deviation compared to the traditional linear risk sharing rule, and this deviation is due to disagreement. Indeed, according to Equations (2.1) and (2.2), the Risk Sharing Rules are given by

$$
y^{*^{i}}=\frac{1}{2} e^{*}+\left(\frac{M^{i}}{M^{1}+M^{2}}-\frac{1}{2}\right) e^{*} .
$$

The first term $\frac{1}{2} e^{*}$ corresponds to the standard linear risk sharing rule. The second term makes the setting with biased beliefs fundamentally different. It is not linear in aggregate endowment. It indicates the deviation from the sharing rule $\frac{1}{2} e^{*}$, i.e., for which states of the world the share of aggregate risk optimally beared by agent $i$ is greater (resp. lower) than $\frac{1}{2}$. The part of aggregate risk optimally beared by agent $i$ is greater than in the standard setting when the subjective belief of agent $i$ is greater than the consensus belief (or equivalently, in our setting with two agents, when the subjective belief of agent $i$ is greater than the subjective belief of the other agent). As already underlined, this is natural since it amounts to saying that the agent bears a larger share of the risk in states of the world that she thinks more probable than the other agent, the good states of the world for Agent 1 and the bad states of the world for Agent 2. The deviation from the standard risk sharing rule is all the more important as the states of the world are extreme.

In the case of logarithmic utility functions, classical tests of efficiency that derive from the seminal work of Mace (1991) are based on the fact that any shock on the aggregate endowment should impact the agents uniformly, i.e. $\frac{y_{t+\Delta t}^{* i}}{y_{t}^{* i}}$ should be the same for all agents. This is the case when the standard risk sharing rule applies, since $\frac{y_{t+\Delta t}^{*^{i}}}{y_{t}^{* i}}$ is then given by $\frac{e_{t+\Delta t}^{*}}{e_{t}^{*}}$. The Efficient Market Hypothesis would be rejected by these tests in our setting. A test of allocational efficiency 
that would be compatible with our setting should then take into account the impact that aggregate shocks have on individual beliefs.

Let us analyze the implications of the risk sharing rules in terms of trading volumes. Equation (6.1) means that, as expected, heterogenous beliefs that are on average rational generate trading volume. Indeed, there is no trading in the standard setting since the optimal demands $\frac{1}{2} e^{*}$ coincide with the initial endowments while in our setting the trading volumes are given by $\left|\left(\frac{M^{1}-M^{2}}{M^{1}+M^{2}}\right) e^{*}\right|$. The trading volumes are almost surely non null, and they are time-varying and stochastic. For example, in states of the world $\omega$ for which $M^{1}(\omega)>M^{2}(\omega)$, the optimal demand of Agent 1 is greater than her initial endowment $\frac{1}{2} e^{*}$, which means that Agent 1 is a net buyer of this state and Agent 2 is a net seller of this state. The trading volumes are increasing in the level of diagreement $\delta$ : the more heterogeneous the beliefs of the agents, the higher the incentives to trade.

Moreover, trading volumes are small when aggregate endowment is close to its average value and trading volumes are large when aggregate endowment is very high, i.e. in very good states of the world, when agents are more wealthy and the economy is dominated by the optimistic agents. This is consistent with the empirical finding that volumes of trade are more important in bull markets. As noted by e.g. Karpoff (1987) or more recently by Heston and Sadka (2005) and Frazzini and Lamont (2007), there is a strong positive contemporaneous relationship between trading activity and stock prices. Most theories ${ }^{15}$ trying to account for this positive relationship rely on short sales constraints, since, as first underlined by Miller (1977) or Harrison and Kreps (1978), differences of opinion under short sales constraints lead to overpricing. We obtain in our setting a similar result without introducing short sales contraints.

Note that the trading volumes in our setting are in fact the same as in a standard economy with two agents with rational beliefs, logarithmic utility functions and heterogeneous endowments, not perfectly correlated with aggregate endowment, given by $e_{i}^{*}=\left(\frac{M^{i}}{M^{1}+M^{2}}\right) e^{*}$. As far as trading volumes are concerned, on average rational biased beliefs have the same impact as endowments heterogeneity. Note that there would be no trading in the economy populated by the same two agents with logarithmic utility functions and endowments $e^{*^{i}}$ but with the

\footnotetext{
${ }^{15}$ Another strand of explanations involve irrational traders. In Hong and Yu (2006), high volume indicates the presence of noise traders, and rational investors demand a risk premium to compensate for the sentiment risk. Other studies rely on the "attention grabbing" hypothesis (Lee, 1992, Barber and Odean, 2004).
} 
same belief given by the consensus belief $Q$. It is beliefs heterogeneity (and not beliefs subjectivity) that generates trading.

\section{Remarks and Extensions}

\subsection{Comparison at the aggregate level with a model with two scenar- ios}

The aim of this section is to compare our setting with the setting of an economy with a single agent who thinks that the aggregate endowment growth rate can be $(\mu+\sigma \delta)$ (with a probability $\frac{1}{2}$ ) forever or $(\mu-\sigma \delta)$ (with a probability $\frac{1}{2}$ ) forever. We shall refer to our unbiased disagreement setting as setting B (beliefs) and to the setting with the scenarios as setting $S^{16}$ and compare the properties of both settings at the aggregate level (comparing the properties at the individual level would be meaningless).

We introduce the random variable $\widetilde{\mu}$ taking the values $(\mu+\sigma \delta)$ and $(\mu-\sigma \delta)$ with equal probabilities. We refer to this random variable as the consensus scenario. As far as asset pricing is concerned, the risk free rate and the market price of risk in Setting $\mathrm{S}$ are given by

$$
\begin{aligned}
r_{t}^{f} & =\widetilde{\mu}-\sigma^{2} \\
M P R_{t} & =M P R(\text { stdd })-\widetilde{\delta}
\end{aligned}
$$

where $\widetilde{\delta} \equiv \frac{1}{\sigma}(\widetilde{\mu}-\mu)$ takes the values $\delta$ and $-\delta$ with equal probabilities. This means that the risk free rate and the market price of risk take the same two possible values with equal probabilities, at all dates $t$, independently of the rest of the economy (in particular, independently from aggregate endowment $\left.e^{*}\right)$. This is very different from Setting B. In particular, none of the dynamic properties obtained in Proposition 1 remains true in Setting S. However, we have previously underlined that asymptotically Setting B approaches in distribution Setting S (see, e.g., Proposition 1.3.). The asymptotic results on the discount rates remain valid. Analogously, it is easy to verify that the distribution of $\log e_{t}^{*}$ for the consensus scenario is a mixture of the distribution of $\log e_{t}^{*}$ under each scenario. Hence the results on the distribution of $e_{t}^{*}$ remain true in Setting $\mathrm{S}$. In case $\mathrm{S}$, we have for all $t, \log e_{t}^{*} \sim \mathcal{N}\left(\tilde{\mu} t, \sigma^{2} t\right)$. However, as in the case of the risk free rate and the market price of risk, the dynamic properties are different.

\footnotetext{
${ }^{16} \mathrm{~A}$ similar setting is considered in e.g. Gollier (2008).
} 
Indeed, in Setting S, we have $d e_{t}^{*}=\widetilde{\mu} e_{t}^{*} d t+\sigma e_{t}^{*} d W_{t}$, which is to be compared to the dynamics $d e_{t}^{*}=\left(\mu+\tau_{1} \delta_{1}+\tau_{2} \delta_{2}\right) e_{t}^{*} d t+\sigma e_{t}^{*} d W_{t}^{Q}$.

To sum up, the two models have in common at the aggregate level the distributional properties of $\log e^{*}$, and the asymptotic price distributions. However, the trajectorial properties, relating prices and the rest of the economy don't remain valid, neither do the dynamic properties. This means that these properties result from the disagreement among agents and cannot be retrieved by the introduction of additional risk or uncertainty.

\subsection{Other Utility Functions}

The aim of this section is to consider if our results are robust to more general utility functions.

We have considered so far logarithmic utility functions. The first reason is analytical tractability. Indeed, "log utility functions are singular in their capacity to cope with heterogeneous beliefs, while not imposing unreasonable restrictions on tastes" (Rubinstein, 1975). The second reason, which is particularly important for our issue, is the fact that it has been shown in the literature on heterogeneous beliefs (see e.g. Jouini and Napp, 2007), that apart in the logarithmic setting there is a bias ${ }^{17}$ induced by beliefs heterogeneity which makes the heterogeneous setting fundamentally different from the homogeneous setting. Since the aim of this paper is to analyse if the heterogeneous setting with on average rational agents can be neglected (with the intuition that it cannot), it seems more consistent to consider as our main model the setting which is the most likely to have no impact.

Consider now the more general setting of power utility functions. The setting is the same as in Section 2, except that the agents' utility function is such that $u^{\prime}(x)=x^{-1 / \eta}$. We consider the case with the same initial endowments. We provide in Appendix B all the results in this setting and we sum up here the main conclusions. As shown in e.g. Jouini and Napp (2007), there is an aggregation bias. However, modulo this bias, the conclusions of the previous sections remain essentially valid. As far as survival issues are concerned, we obtain that none of the agents vanishes, both agents survive and there is a sort of stationarity (see Appendix B, I-B). As in the logarithmic setting, in the long

\footnotetext{
${ }^{17}$ Moreover, the biases are in opposite directions depending on the position of the level of risk aversion with respect to 1 , which makes the log-utility setting central in the analysis of beliefs heterogeneity, .
} 
run agents share out the states of the world, the optimistic agent dominating the economy in the good states of the world and the pessimistic agent dominating the economy in the bad states of the world. At each date $t$, there is a pessimistic bias in bad states of the world and an optimistic bias in good states of the world (Appendix B, I-C). As far as asset pricing results are concerned, we obtain (Appendix B, I-D) that the market price of risk is countercyclical and exhibits mean reversion as well as short run momentum. The risk free rate is procyclical. The yield curve is still decreasing in the long run but its short term behavior depends upon $\eta$. The conclusions on the risk sharing rule and trading volumes remain the same as in the logarithmic setting (Appendix B, I-F). Moreover, we obtain in the power utility setting results on the volatility of the stock whose associated dividends are the aggregate endowment (Appendix B, I-G). In the myopic logarithmic setting, this volatility is necessarily given by the volatility of aggregate consumption, as in the standard rational setting. For more general power functions, it is time and state varying. It is equal to the volatility of the economy made of the pessimistic agents only in very bad states of the world and to the volatility of the economy made of the optimistic agents only in very good states of the world. In the long run, the volatility converges to the volatility of aggregate consumption. The stock price dividend ratio $\frac{S_{t}}{e_{t}^{*}}$ converges in the long run with the same probability either to the price dividend ratio of the pessimistic economy or to the price dividend ratio of the optimistic economy.

Note that our results are not robust to an exponential specification of the utility function, in particular those on asset pricing. According to Jouini and Napp (2007, Section 4.1), the market price of risk in the exponential setting is given by the standard market price of risk $M P R_{t}=M P R(s t d d)$ and the risk free rate is given by the standard risk free rate modulo a (constant) bias due to beliefs dispersion, $r_{t}^{f}=r_{t}^{f}(\operatorname{stdd})+\frac{1}{2} \delta^{2}$. There is no state nor time dependence. In fact, in the exponential setting, as in the logarithmic or power setting, the risk free rate and the market price of risk are given by the risk tolerance weighted averages of the individual risk free rates and market prices of risk (modulo the bias). In the case of exponential utility functions (CARA), the relative levels of absolute risk tolerances are constant and lead to constant market price of risk and risk free rate. In the case of logarithmic or power utility functions the (relative) levels of absolute risk tolerances are given by the consumption shares, which are time varying and stochastic. This induces time varying and stochastic market price of risk and risk free rate. It is important to emphasize that the fluctuations in the market price of risk and in the risk free rate are due to 
fluctuations in the levels of risk tolerances (and not in the levels of consumptions shares, even if both notions coincide in the case of power utility functions).

\subsection{Models with Irrational as well as Rational Agents}

The aim of this section is to consider to what extent our results on irrational traders are robust to the presence of rational traders on the markets. For this purpose, we consider a model that is analogous to the model of Section 2 except that there are now three agents : Agent 1, as in Section 2, overestimating the instantaneous expected growth rate by $\sigma \delta$, Agent 2, as in Section 2, underestimating the instantaneous expected growth rate by $\sigma \delta$ and Agent 3, rationally expecting the instantaneous growth rate $\mu$. We suppose that the three agents have the same initial endowment $(1 / 3) e^{*}$. Adopting the same notations as in Section 3 , we easily get in this setting that the individual consumptions shares are given by $\tau_{i} \equiv \frac{y^{*^{i}}}{e^{*}}=\frac{1}{3} \frac{M^{i}}{M^{1}+M^{2}+1}$, where $M^{3} \equiv 1$.

The survival properties are different in this setting. Indeed, as shown in Yan (2008a), it is easy to obtain in this setting that $\tau_{3}(t)$ converges to 1 almost surely, i.e. only the rational agent survives ${ }^{18}$. This implies that instead of converging to an economy with two possible scenarii (one pessimistic scenario and one optimistic scenario), the economy converges to an economy with the rational scenario only. In particular, the instantaneous risk free rate converges to the rational risk free rate and the market price of risk converges to the standard market price of risk. However, as shown in Yan (2008a), the selection process can be very slow.

The other results remain essentially true. The consumption shares $\tau_{1}(t)$ and $\tau_{2}(t)$ have the same distribution for all $t$, and none of the irrational agents wins. The instantaneous prices are on average given by the rational prices, i.e. $E\left[M P R_{t}\right]=M P R\left(\right.$ stdd) and $E\left[r_{t}^{f}\right]=r^{f}$ (stdd). However, we get as in Section 3 that $\frac{\tau_{1}(t)}{\tau_{2}(t)}=\exp \left(2 \delta W_{t}\right)$, hence $\tau_{1}>\tau_{2}$ if and only if $\log e_{t}^{*}>E\left[\log e_{t}^{*}\right]$, i.e. in good states of the world. This means that there is an optimistic bias (in terms of consumptions or risk shares) in the good states of the world and a pessimistic bias in the bad states of the world. We still get that the economy is dominated by the pessimistic agents in the very bad states of the world and dominated by the optimistic agents in the very good states of the world. The

\footnotetext{
${ }^{18}$ We have for $i=1,2, M_{t}^{i}=\exp \left[t\left(\delta_{i} \frac{W_{t}}{t}-\frac{\delta_{i}^{2}}{2}\right)\right]$ which converges to 0 when $t$ increases, since $\frac{W_{t}}{t} \rightarrow_{t \rightarrow \infty} 0$ a.s.
} 
market price of risk, which is given by $M P R_{t}=M P R($ stdd $)-\left(\tau_{1}(t)-\tau_{2}(t)\right) \delta$, is countercyclical and exhibits momentum. The risk free rate, which is given by $r_{t}^{f}=r^{f}$ (stdd) $+\sigma\left(\tau_{1}(t)-\tau_{2}(t)\right) \delta$ is procyclical. The yield curve is still decreasing and converging to the discount rate of the more pessimistic agent, i.e. Agent 2 (even though the risk free rate converges to the rational rate). The consensus belief is still a mixture of the individual beliefs, which generates more variance, leaving the mean unchanged. The results on the risk sharing rule and the volumes remain exactly the same as in Section 6 .

\subsection{Other Models of Disagreement}

We have considered so far a model of disagreement, in which the disagreement is on average zero but also constant in time and in states of the world, i.e. $\delta_{i} \in \mathbb{R}$. We can consider more general specifications of disagreement.

Consider the setting of Section 2 except that we assume that $d M_{t}^{i}=\delta_{i} M_{t}^{i} d W_{t}$, where $\delta_{i}$ is nomore a constant but a stochastic process $\left(\delta_{i}(t, \omega)\right)$. This is roughly the most general model of beliefs divergence in a diffusion setting ${ }^{19}$. In this model, an agent is irrational at time $t$ and in state $\omega$, if $\delta_{i}(t, \omega) \neq 0$, optimistic if $\delta_{i}(t, \omega)>0$ and pessimistic if $\delta_{i}(t, \omega)<0$. In particular, we may consider models in which an agent is sometimes optimistic and sometimes pessimistic (the sign of the stochastic process $\left(\delta_{i}(t, \omega)\right)$ is not constant). We assume that the agents are on average rational, i.e. that $\delta_{1}(t, \omega)=\delta(t, \omega)$ and $\delta_{2}(t, \omega)=-\delta(t, \omega)$.

In such a setting the consumption shares are given by $\tau_{i}(t, \omega)=\frac{1}{2} \frac{M^{i}}{M^{1}+M^{2}}$, which means that, as before, the consumption share is high in states of the world that the agent thinks more probable. The consumption share $\tau_{1}(t, \omega)$ of Agent 1 is greater than the consumption share $\tau_{2}(t, \omega)$ of Agent 2 in states $\omega$ that she overweights, more precisely, in states $\omega$ such that $\int_{0}^{t} \delta(s, \omega) d W_{s}>0$. Note that on average we have $E\left[\int_{0}^{t} \delta(s, \omega) d W_{s}\right]=0$. This quantity $\int_{0}^{t} \delta(s, \omega) d W_{s}$ in a way measures the degree at which nature has favoured (or disfavoured) Agent 1 with respect to Agent 2 between date 0 and date $t$. In other words there is a bias towards the agent who has been less wrong between date 0 and date $t$, given the evolution of the economy during the same period. We can show (see Appendix B) that, as in Section 3, the consumption share of Agent 1 satisfies

\footnotetext{
${ }^{19}$ Indeed, if $M^{i}$ is a positive martingale process, the fact that it can be written in the form $d M_{t}^{i}=\delta_{i} M_{t}^{i} d W_{t}$ for some adapted process $\left(\delta_{i}\right)$ is then just a regularity assumption.
} 
the following SDE

$$
d \tau_{1}(t)=2 \delta \tau_{1} \tau_{2}\left[\delta\left(\tau_{2}-\tau_{1}\right) d t+d W_{t}\right]
$$

There is then mean reversion in the dynamics of the consumption shares. Moreover, we have $d \log \left(\frac{\tau_{1}(t, \omega)}{\tau_{2}(t, \omega)}\right)=\delta(t, \omega) d W_{t}$, which means that there is locally a shift in favor of (against) optimistic agents following good (bad) news.

As far as asset pricing issues are concerned, it is easy to obtain that we have

$$
\begin{aligned}
r_{t}^{f} & =r^{f}(\operatorname{stdd})+\sigma \delta(t, \omega)\left[\tau_{1}(t, \omega)-\tau_{2}(t, \omega)\right], \\
M P R_{t} & =M P R(\operatorname{stdd})-\left[\tau_{1}(t, \omega)-\tau_{2}(t, \omega)\right] \delta(t, \omega) .
\end{aligned}
$$

The market price of risk exhibits momentum. The states of the world for which the market price of risk is high are the states of the world for which the risk free rate is low. The market price of risk (resp. the risk free rate) is lower (resp. higher) in states of the world that are "good" for the (locally) optimistic agent (in the sense that her consumption share is high). These are not necessarily the "good" states of the world, since the market price of risk is lower than the standard market price of risk when $\left(\int_{0}^{t} \delta(s, \omega) d W_{s}\right) \delta(t, \omega)>0$.

Notice that after a good shock $\left(\int_{0}^{t} \delta_{i}(s, \omega) d W_{s}\right)$ increases for the optimistic agent leading to a decrease of the market price of risk. There is then a shift towards lower (resp. higher) market prices of risk following good (bad) news. Similarly, there is a shift towards lower (resp. higher) risk free rates following bad (good) news. This means that in the general setting, we retrieve locally the same type of results as in Proposition 1, i.e. the fact that good news decrease the market price of risk and increase the risk free rate.

As in Section 6, we obtain that there is a deviation from the standard linear risk sharing rule and that disagreement generates trading. In particular, the trading volumes are important in good states of the world when in addition $\int_{0}^{t} \delta(s, \omega) d W_{s}$ is small, i.e., when none of the agents have particularly benefitted from their irrationality. Furthermore, we obtain that the discount rate is decreasing in the long run.

To illustrate our results, let us construct a model in which the agents are on average rational, but in which agents can switch from optimism to pessimism and conversely. To make it simple, let us assume that the switches occur at deterministic and regular dates. We take $\delta(s, \omega)=1_{[2 n, 2 n+1[}(s)$ and $\delta(s, \omega)=$ $-1_{[2 n+1,2 n+2[}(s)$. This means that Agent 1 is optimistic for $t \in[0,1[,[2,3[,[4,5[$, 
etc. and pessimistic for $t \in[1,2[,[3,4[,[5,6[$, etc. At each date, the consumption shares $\tau_{1}(t)$ and $\tau_{2}(t)$ have the same distribution and the market price of risk and the risk free rate are on average (over the states of the world) given by the standard quantities. A positive shock leads to an average market price of risk (resp. risk free rate) that is lower (resp. higher) than the standard one. Furthermore, in good states of the world, we have $P\left[\tau_{1}(t, \omega)>\tau_{2}(t, \omega)\right]>1 / 2$ when Agent 1 is the optimistic agent, $P\left[\tau_{2}(t, \omega)>\tau_{1}(t, \omega)\right]>1 / 2$ when Agent 2 is the optimistic agent, which implies that there is an optimistic bias in the good states of the world. This leads to a countercyclical market price of risk and a procyclical risk free rate.

\section{Conclusion}

In this paper we study the impact on the behavior of financial markets of irrational traders, when they are on average rational. To sum up, the model with on average zero disagreement is very different from the standard rational model, although they share common features. As in the standard setting, all agents survive. Moreover, at all date, the consumption shares remain equally distributed. Finally, at all date, the prices remain on average the same as in the standard rational setting. However, the main features are the following.

- Time and state varying market price of risk and risk free rate.

- Countercylical market price of risk (higher in recessions and lower in expansions) and procyclical risk free rate.

- Momentum and mean reverting market price of risk and risk free rate.

- Decreasing yield curve (at least in the long run).

- Possibly higher long run risky asset's return and higher long term risk premia.

- More variance.

- State price densities that are mixtures of lognormal distributions.

- Possibly time and state varying volatility.

- Time and state varying risk sharing rules. 
- Increased trading volumes. More important trading volumes in bull markets.

We emphasize that the obtained properties only result from the (absolute) risk tolerance fluctuations and not on specific learning dynamics. Note also that we do not impose short sales constraints.

\section{References}

[1] Abel, A., 1989. Asset Prices under heterogeneous beliefs: implications for the equity premium. Mimeo, University of Pennsylvania.

[2] Admati, A.R., 1985. A Noisy Rational Expectations Equilibrium for MultiAsset Securities Markets. Econometrica, 53, 629-657.

[3] Aït-Sahalia, Y., and A.W. Lo, 1998. Nonparametric Estimation of State Price Densities Implicit in Financial Asset Prices. Journal of Finance, 53, 499-547.

[4] Barber, B. and T. Odean, 2004. All that Glitters: the Effect of Attention and News on the Buying Behavior of Individual and Institutional Investors. Working Paper.

[5] Basak, S., 2000. A model of dynamic equilibrium asset pricing with heterogeneous beliefs and extraneous risk. Journal of Economic Dynamics and Control, 24, 63-95.

[6] Berrada, T., 2006. Incomplete Information, Heterogeneity and Asset Pricing. Journal of Financial Econometrics, 4, 136-160.

[7] Biais, B., Bossaerts, P. and C. Spatt, 2004. Equilibrium Asset Pricing under Heterogeneous Information, EFA 2004 Maastricht Paper No 5083.

[8] Blanchard, O. and M. Watson, 1986. Are Business Cycles All Alike ? In Robert Gordon, ed. The American Business Cycle, 123-182. Chicago, University of Chicago Press.

[9] Blume, L., and D. Easley, 2006. If You're So Smart, Why Aren't You Rich ? Belief Selection in Complete and Incomplete Markets. Econometrica, 74, 929-966. 
[10] Cabrales, A., and T. Hoshi, 1996. Heterogeneous Beliefs, Wealth Accumulation, and Asset Price Dynamics. Journal of Economic Dynamics and Control, 20, 1073-1100.

[11] Calvet, L., Grandmont, J.-M., and I. Lemaire, 2002. Aggregation of Heterogeneous Beliefs and Asset Pricing in Complete Financial Markets. Working Paper.

[12] Campbell J.Y. and J.H. Cochrane, 1999. By Force of Habit: A Consumption-Based Explanation of Aggregate Stock Market Behavior. Journal of Political Economy, 107, 205-251.

[13] Chateaunef, A., and M. Cohen, 1994. Risk Seeking with Diminishing Marginal Utility in a Nonexpected Utility Model. Journal of Risk and Insurance, 9, 77-91.

[14] David, A., 2008. Heterogeneous Beliefs, Speculation, and the Equity Premium. Journal of Finance, 63, 41-83.

[15] David, A., and P. Veronesi, 2002. Option Prices with Uncertain Fundamentals. Working Paper.

[16] DeMarzo, P. and C. Skiadas, 1998. Aggregation, Determinacy, and Informational Efficiency for a Class of Economies with Asymetric Information. Journal of Economic Theory, 80, 123-152.

[17] Detemple J., and S. Murthy, 1994. Intertemporal Asset Pricing with Heterogeneous Beliefs. Journal of Economic Theory, 62, 294-320.

[18] Diecidue, E., and P. Wakker, 2001. On the Intuition of Rank Dependent Utility. Journal of Risk and Insurance, 281-298.

[19] Duchin, R. and M. Levy, 2008. Disagreement, Portfolio Optimization and Excess Volatility. Working paper.

[20] Dumas, B., Kurshev A., and R. Uppal, 2009. Equilibrium Portfolio Strategies in the Presence of Sentiment Risk and Excess Volatility. To appear, Journal of Finance.

[21] Fama, E. and K. French, 1988. Dividend yields and expected stock returns. Journal of Financial Economics, 22, 3-25. 
[22] Fama, E. and K. French, 2006. The Behavior of Interest Rates. Review of Financial Studies, 19, 2, 359-379.

[23] Fama, E. and K. French, 2008. Disagreement, Tastes and Asset Prices. CRSP Working Paper No. 552

[24] Frazzini, A. and O. Lamont, 2007. Earnings announcement premium and trading volume. NBER Working paper 13090. http://www.nber.org/papers/w13090

[25] Friedman, B., 1986. Money, Credit and Interest Rates in the Business Cycle. In Robert Gordon, ed. The American Business Cycle, 123-182. Chicago, University of Chicago Press.

[26] Friedman, M., 1953. The case for flexible exchange rates. Essays in Positive Economics, University of Chicago Press, Chicago.

[27] Gollier, C., 2007. Whom Should We Believe ? Aggregation and Heterogeneous Beliefs. Journal of Risk and Uncertainty, 35, 107-127.

[28] Gollier, C., 2008. Discounting with Fat-Tailed Economic Growth. Journal of Risk and Uncertainty, 37, 171-186.

[29] Harrison, J.M., and D.M. Kreps, 1978. Speculative Investor Behavior in a Stock Market with Heterogeneous Expectations. Quarterly Journal of Economics, 93, 323-336.

[30] Heston, S., and R. Sadka, 2005. Seasonal Liquidity and Stock Returns. Working paper.

[31] Hong, H., and J. Yu, 2006. Gone Fishin': Seasonality in Trading Activity and Asset Prices. Working Paper.

[32] Hong, H. and J. Stein, 2007. Disagreement and the Stock Market. Journal of Economic Perspectives, 21, 109-128.

[33] Jackwerth, J.C., and M. Rubinstein, 1996. Recovering Probability Distributions from Asset Prices. Journal of Finance, 51, 1611-1631.

[34] Jegadeesh, N., and S. Titman, 1993. Returns to buying winners and selling losers: Implications for market Efficiency. Journal of Finance, 48, 93-130. 
[35] Jouini, E. , and C. Napp, 2007. Consensus Consumer and Intertemporal Asset pricing with Heterogeneous Beliefs. Review of Economic Studies, 74, 1149-1174.

[36] Jouini, E. , Marin, J.-M. and C. Napp, 2008a. Discounting and Divergence of Opinion. Working paper.

[37] Jouini, E. and C. Napp, 2008b. Behavioral Properties of the Representative Agent. Working Paper.

[38] Karpoff, J., 1987. The Relation between Price Change and Trading Volume: a Survey. Journal of Financial and Quantitative Analysis, 22, 109-126.

[39] Kogan, L., Ross, S., Wang, J., and M. Westerfield, 2006. The Price Impact and Survival of Irrational Traders. Journal of Finance, 61, 195-229.

[40] Kogan, L., Ross, S., Wang, J., and M. Westerfield, 2008. Market Selection. Working Paper.

[41] Lee, C.M.C, 1992. Earnings News and Small Traders: an Intraday Analysis. Journal of Accounting and Economics, 15, 265-302.

[42] Levy, H., Levy, M. and G. Benita, 2006. Capital Asset Prices with Heterogeneous Beliefs. Journal of Business, 79, 1317-1354.

[43] Li, T., 2007. Heterogeneous Beliefs, Asset Prices, and Volatility in a Pure Exchange Economy. Journal of Economic Dynamics and Control, 31, 16971727.

[44] Mace, B. J., 1991. Full Insurance in the Presence of Aggregate Uncertainty. Journal of Political Economy, 99, 928-956.

[45] Merton, R., 1980. On Estimating the Expected Return on the Market: an Exploratory Investigation. Journal of Financial Economics, 8, 323-362.

[46] Miller, E. M., 1977. Risk, Uncertainty, and Divergence of Opinion. Journal of Finance, 32, 1151-1168.

[47] Rubinstein, M., 1975. The Strong Case for the Generalized Logarithmic Utility Model as The Premier Model of Financial Markets. The Journal of Finance, 31, 551-571. 
[48] Sandroni, A., 2000. Do Markets Favor Agents Able to Make Accurate Predictions ? Econometrica, 68, 1303-1334.

[49] Scheinkman, J., and W. Xiong, 2003. Overconfidence and Speculative Bubbles. Journal of Political Economy, 111, 1183-1219.

[50] Yaari, 1987. The Dual Theory of Choice under Risk. Econometrica, 55, 95-115.

[51] Yan, H., 2008a. Natural Selection in Financial Markets: Does It Work ? Management Science, 54, 1935-1950.

[52] Yan, H., 2008b. Is noise trading cancelled out by aggregation? Working Paper, Yale School of Management.

[53] Zapatero, F., 1998. Effects of Financial Innovations on Market Volatility when Beliefs are Heterogeneous. Journal of Economic Dynamics and Control, 22, 597-626. 


\section{APPENDIX A}

\section{Proof of Proposition 1}

1. and 2. According to Equation (2.1), we have $\tau_{1}=\frac{\left(M_{t}^{1} / M_{t}^{2}\right)}{1+\left(M_{t}^{1} / M_{t}^{2}\right)}$. We have $M_{t}^{1}=\exp \left(\delta W_{t}-\frac{1}{2} \delta^{2} t\right)$ and $M_{t}^{2}=\exp \left(-\delta W_{t}-\frac{1}{2} \delta^{2} t\right)$, hence $\tau_{1}(t)=\frac{\left(\exp 2 \delta W_{t}\right)}{1+\left(\exp 2 \delta W_{t}\right)}$ and $\tau_{2}(t)=\frac{\left(\exp -2 \delta W_{t}\right)}{1+\left(\exp -2 \delta W_{t}\right)}$ with $W_{t} \sim \mathcal{N}(0, t)$. The consumption shares are then equally distributed. Elementary algebra gives the expression of the densities $f_{\tau_{1}(t)}$ and $f_{\tau_{2}(t)}$. Indeed, the distribution of $\frac{\exp X}{1+\exp X}$ for $X \sim \mathcal{N}\left(0, \sigma^{2}\right)$ has the density $g\left(\frac{x}{1-x}\right) \frac{1}{(1-x)^{2}} 1_{[0,1]}(x)$, where $g$ is the density of a lognormal distribution $\ln \mathcal{N}\left(0, \sigma^{2}\right)$. Taking $\sigma^{2}=4 \delta^{2} t$ leads to $f_{\tau_{1}(t)}(x)=\frac{1}{2 \sqrt{2 \pi t} \delta} \frac{1}{x(1-x)} \exp -\frac{1}{2} \frac{\left[\log \left(\frac{x}{1-x}\right)\right]^{2}}{4 \delta^{2} t} \mathbf{1}_{[0,1]}(x)$. This distribution is symmetric with respect to $1 / 2$; in particular, we have $E\left[\tau_{i}(t)\right]=1 / 2$. 3. For $\varepsilon>0$, it is immediate that $f_{\tau_{i}(t)}(x)$ decreases with $t$ and converges uniformly to 0 on $[\varepsilon, 1-\varepsilon]$. The distribution being symmetric, the result follows.

\section{Proof of Proposition 2}

1. and 2. According to Equation (2.1), we have $\tau_{i}=\frac{1}{2} \frac{M^{i}}{M}$. The optimal consumption shares are then time varying and stochastic. Agent $i$ has a larger share of aggregate consumption in states of the world for which $M^{i}$ is large compared to $M$, i.e. the states of the world that she overweights. 3. Since $\tau_{1}(t)=\frac{\exp \left(2 \delta W_{t}\right)}{1+\exp \left(2 \delta W_{t}\right)}$ and $\tau_{2}(t)=\frac{1}{1+\exp \left(2 \delta W_{t}\right)}$, we have $\tau_{1}>\tau_{2}$ if and only if $\exp \left(2 \delta W_{t}\right)>1$, i.e. if and only if $W_{t}>0$. Since $\tau_{1}(t)=f\left(W_{t}\right)$ with $f(x)=$ $\frac{\exp (2 \delta x)}{1+\exp (2 \delta x)}$, it is immediate to get that $f$ is increasing and that there is a shift towards optimistic agents following good news. 4. Since $\tau_{1}(t)=\frac{\exp \left(2 \delta W_{t}\right)}{1+\exp \left(2 \delta W_{t}\right)}$, the share $\tau_{1}$ is near zero when $W$ takes very high negative values, and it is near one when $W$ takes very high positive values. 5 . We have $\tau_{1}(t)=f\left(W_{t}\right)$ with $f(x)=\frac{\exp (2 \delta x)}{1+\exp (2 \delta x)}$. Itô's Lemma and elementary algebra give the following Stochastic Differential Equations

$$
\begin{aligned}
d \tau_{1}(t) & =2 \delta^{2} \tau_{1}\left(1-\tau_{1}\right)\left(1-2 \tau_{1}\right) d t+2 \delta \tau_{1}\left(1-\tau_{1}\right) d W_{t} \\
& =2 \delta \tau_{1} \tau_{2}\left[\delta\left(\tau_{2}-\tau_{1}\right) d t+d W_{t}\right] \\
d \tau_{2}(t) & =-2 \delta \tau_{1} \tau_{2}\left[\delta\left(\tau_{2}-\tau_{1}\right) d t+d W_{t}\right]
\end{aligned}
$$

It is immediate that the drift of $\tau_{1}$ is positive for $\tau_{1}<\frac{1}{2}$ and negative for $\tau_{1}>\frac{1}{2}$. The process $\tau_{1}$ exhibits then mean reversion around $\frac{1}{2}$.

Proof of Proposition 1 
1. Since $q^{*}=\left(\frac{1}{2} M^{1}+\frac{1}{2} M^{2}\right)\left(e^{*}\right)^{-1}, r_{f}=-\mu_{q^{*}}$ and $M P R=-\sigma_{q^{*}}$, we obtain through Ito's Lemma

$$
\begin{aligned}
r_{t}^{f} & =\mu-\sigma^{2}+\sigma\left(\tau_{1}(t) \delta-\tau_{2}(t) \delta\right), \\
M P R_{t} & =\sigma-\left(\tau_{1}(t)-\tau_{2}(t)\right) \delta .
\end{aligned}
$$

2. The density of the random variable $\left(\tau_{1}-\tau_{2}\right)$ is given by $f_{\left(\tau_{1}-\tau_{2}\right)}(x)=$ $\frac{1}{2} f_{\tau_{1}}\left(\frac{x+1}{2}\right) 1_{[-1 ; 1]}(x)$. Since $f_{\tau_{1}}$ is symmetric with respect to $\frac{1}{2}$, it is immediate that $f_{\left(\tau_{1}-\tau_{2}\right)}$ is symmetric with respect to 0 , and we easily get $E\left[r_{t}^{f}\right]=\mu-\sigma^{2}$ and $E\left[M P R_{t}\right]=\sigma$. 3. According to Equation (4.2), the market price of risk is lower in states of the world where $\tau_{1}(t)>\tau_{2}(t)$ and decreases with $\tau_{1}(t)$. Proposition 2 concludes. 4. According to Equation (4.1), the risk free rate is higher in states of the world where $\tau_{1}(t)>\tau_{2}(t)$ and increases with $\tau_{1}(t)$. Proposition 2 concludes. 5. This is a consequence of Equation 4.2 and of the continuity of $\tau_{1}(t)$. 6. This is a direct consequence of Equations (4.1) and (4.2) and of Point 5 of Proposition 2.

\section{Proof of Proposition 2}

We can apply Jouini-Napp (2008) to our specific setting by taking $\rho^{i}=\rho$, $\delta_{1}=\delta$ and $\delta_{2}=-\delta$. We rederive this result here for the sake of completeness. We have

$$
R_{t}=-\frac{1}{t} \log E\left[\left(\frac{1}{2} M_{t}^{1}+\frac{1}{2} M_{t}^{2}\right) \exp (-\rho t)\left(e_{t}^{*}\right)^{-1}\right] .
$$

We then have $R_{t}=\rho+\mu-\sigma^{2}-\frac{1}{t} \log E\left[\frac{1}{2} \exp \left((\delta-\sigma) W_{t}-\frac{\delta^{2}+\sigma^{2}}{2} t\right)+\frac{1}{2} \exp \left((-\delta-\sigma) W_{t}-\frac{\delta^{2}+\sigma^{2}}{2} t\right)\right]$, hence $R_{t}=\rho+\mu-\sigma^{2}-\frac{1}{t} \log \left[\frac{1}{2} \exp (-\delta \sigma t)+\frac{1}{2} \exp (+\delta \sigma t)\right]$. The rest is immediate.

\section{Proof of Proposition 3}

Let $\mu_{S}(s t d d) \equiv \rho+\mu-\sigma^{2}+\sigma \sigma_{S}$. We have $\mu_{S}(t)=\mu_{S}(s t d d)+\delta\left(\sigma-\sigma_{S}\right)\left(\tau_{1}-\tau_{2}\right)$ and

$$
\frac{1}{T} \log E\left[S_{T}\right]=\frac{1}{T} \log E\left[\exp \left\{\int_{0}^{T}\left(\mu_{S}(t)-\frac{\sigma_{S}^{2}}{2}\right) d t+\sigma_{S} W_{T}\right\}\right] .
$$

1. Let us first prove that $\frac{1}{T} \log E\left[S_{T}\right] \leq \mu_{S}(s t d d)+\delta(\sigma-\sigma S)$. Since by hypothesis $\delta\left(\sigma-\sigma_{S}\right) \geq 0$, we have $\mu_{S}(t) \leq \mu_{S}(s t d d)+\delta\left(\sigma-\sigma_{S}\right)$, hence

$$
\begin{aligned}
\frac{1}{T} \log E\left[S_{T}\right] & \leq \frac{1}{T} \log E\left[\exp \left\{\left(\int_{0}^{T} \mu_{S}(s t d d)+\delta\left(\sigma-\sigma_{S}\right)-\frac{\sigma_{S}^{2}}{2}\right) d t+\sigma_{S} W_{T}\right\}\right] \\
& \leq \mu_{S}(s t d d)+\delta\left(\sigma-\sigma_{S}\right) .
\end{aligned}
$$


2. We have by Equation (8.1)

$$
\begin{aligned}
\frac{1}{T} \log E\left[S_{T}\right] & =\mu_{S}(s t d d)+\frac{1}{T} \log E\left[\exp \left\{\int_{0}^{T}\left(\delta\left(\sigma-\sigma_{S}\right)\left(\tau_{1}-\tau_{2}\right)-\frac{\sigma_{S}^{2}}{2}\right) d t+\sigma_{S} W_{T}\right\}\right] \\
& =\mu_{S}(s t d d)+\frac{1}{T} \log E\left[\exp \left\{\int_{0}^{T}\left(\delta\left(\sigma-\sigma_{S}\right) \frac{\exp \left(2 \delta W_{t}\right)-1}{\exp \left(2 \delta W_{t}\right)+1}-\frac{\sigma_{S}^{2}}{2}\right) d t+\sigma_{S} W_{T}\right\}\right]
\end{aligned}
$$

We introduce the probability measure $\bar{P}$ such that $\left.\frac{d \bar{P}}{d P}\right|_{F_{t}}=\exp \left(\sigma_{S} W_{t}-\frac{\sigma_{S}^{2}}{2} t\right)$. We have

$$
\frac{1}{T} \log E\left[S_{T}\right]=\mu_{S}(s t d d)+\frac{1}{T} \log E^{\bar{P}}\left[\exp \left\{\int_{0}^{T}\left(\delta\left(\sigma-\sigma_{S}\right) \frac{\exp \left(2 \delta W_{t}\right)-1}{\exp \left(2 \delta W_{t}\right)+1}\right) d t\right\}\right]
$$

Letting $\bar{W}_{t} \equiv W-\sigma_{S} t$, we know that $\bar{W}$ is a Brownian motion under $\bar{P}$ and we obtain

$$
\begin{aligned}
\frac{1}{T} \log E\left[S_{T}\right] & =\mu_{S}(s t d d)+\frac{1}{T} \log E^{\bar{P}}\left[\exp \left\{\int_{0}^{T}\left(\delta\left(\sigma-\sigma_{S}\right) \frac{\exp \left(2 \delta \bar{W}_{t}+2 \delta \sigma_{S} t\right)-1}{\exp \left(2 \delta \bar{W}_{t}+2 \delta \sigma_{S} t\right)+1}\right) d t\right\}\right] \\
& =\mu_{S}(s t d d)+\frac{1}{T} \log E\left[\exp \left\{\int_{0}^{T}\left(\delta\left(\sigma-\sigma_{S}\right) \frac{\exp \left(2 \delta W_{t}+2 \delta \sigma_{S} t\right)-1}{\exp \left(2 \delta W_{t}+2 \delta \sigma_{S} t\right)+1}\right) d t\right\}\right] .
\end{aligned}
$$

We let $Y_{t} \equiv \frac{\exp \left(2 \delta W_{t}+2 \delta \sigma_{S} t\right)-1}{\exp \left(2 \delta W_{t}+2 \delta \sigma_{S} t\right)+1}$. We want to show that $\lim \inf \frac{1}{T} \log E\left[S_{T}\right] \geq \mu_{S}(s t d d)+$ $\delta\left(\sigma-\sigma_{S}\right)$ or equivalently that $\liminf E\left[\exp \left\{\delta\left(\sigma-\sigma_{S}\right) N\left(\frac{1}{N} \int_{0}^{N} Y_{t} d t\right)\right\}\right]^{1 / N} \geq$ $\exp \left(\delta\left(\sigma-\sigma_{S}\right)\right)$. By Hölder Inequality, we have $E\left[\exp \left\{\delta\left(\sigma-\sigma_{S}\right) N\left(\frac{1}{N} \int_{0}^{N} Y_{t} d t\right)\right\}\right]^{1 / N} \geq$ $E\left[\exp \left\{\delta\left(\sigma-\sigma_{S}\right)\left(\frac{1}{N} \int_{0}^{N} Y_{t} d t\right)\right\}\right]$. The quantity $Y_{t}=\frac{\exp t\left(2 \delta \frac{W_{t}}{t}+2 \delta \sigma_{S}\right)-1}{\exp t\left(2 \delta \frac{W_{t}}{t}+2 \delta \sigma_{S}\right)+1}$ converges almost surely towards 1 since $\frac{W_{t}}{t}$ converges towards 0 almost surely. Furthermore $Y_{t}$ is bounded. By Césaro's Lemma, we have that $\frac{1}{N} \int_{0}^{N} Y_{t} d t$ also converges almost surely towards 1 , hence $\exp \left\{\delta\left(\sigma-\sigma_{S}\right)\left(\frac{1}{N} \int_{0}^{N} Y_{t} d t\right)\right\}$ converges almost surely to $\exp \left(\delta\left(\sigma-\sigma_{S}\right)\right)$. Now, by Fatou's Lemma, we have

$\liminf E\left[\exp \left\{\delta\left(\sigma-\sigma_{S}\right)\left(\frac{1}{N} \int_{0}^{N} Y_{t} d t\right)\right\}\right] \geq E\left[\liminf \exp \left\{\delta\left(\sigma-\sigma_{S}\right)\left(\frac{1}{N} \int_{0}^{N} Y_{t} d t\right)\right\}\right]$ hence $\lim \inf E\left[\exp \left\{\delta\left(\sigma-\sigma_{S}\right)\left(\frac{1}{N} \int_{0}^{N} Y_{t} d t\right)\right\}\right] \geq \exp \left(\delta\left(\sigma-\sigma_{S}\right)\right)$ and $\liminf \frac{1}{T} \log E\left[S_{T}\right] \geq$ $\mu_{S}(s t d d)+\delta\left(\sigma-\sigma_{S}\right)$. 
Proof of Corollary 4

Immediate according to Propositions 2 and 3.

\section{APPENDIX B}

\section{I - Results for Power Utility Functions}

\section{A. Existence Result}

For general $\eta$, if $(1-\eta)(\mu-\sigma \delta)-\frac{1}{2} \frac{1}{\eta}(1-\eta) \sigma^{2}>0$, then there exists an equilibrium $\left(q^{*},\left(y^{*^{i}}\right)_{i=1,2}\right)$ given by

$$
\begin{aligned}
y_{1}^{*} & =\left(\frac{\gamma_{1}\left(M^{1}\right)^{\eta}}{\gamma_{1}\left(M^{1}\right)^{\eta}+\gamma_{2}\left(M^{2}\right)^{\eta}}\right) e^{*}, \quad y_{2}^{*}=\left(\frac{\gamma_{2}\left(M^{2}\right)^{\eta}}{\gamma_{1}\left(M^{1}\right)^{\eta}+\gamma_{2}\left(M^{2}\right)^{\eta}}\right) e^{*} \\
q^{*} & =\left(\gamma_{1}\left(M^{1}\right)^{\eta}+\gamma_{2}\left(M^{2}\right)^{\eta}\right)^{1 / \eta}\left(e^{*}\right)^{-1 / \eta}
\end{aligned}
$$

for some positive constants $\left(\gamma_{i}\right)$ with $\gamma_{1}+\gamma_{2}=1$. For $\eta=1 / 2$, we have $\frac{\gamma_{1}}{\gamma_{2}}=$ $\sqrt{\frac{\mu-\sigma^{2}+\sigma \delta}{\mu-\sigma^{2}-\sigma \delta}}$. More generally, for $\eta<1$, there is a small bias towards the optimistic investor $\left(\gamma_{1}>1 / 2\right)$, and for $\eta>1$, there is a small bias towards the pessimistic investor $\left(\gamma_{2}>1 / 2\right)$.

B. Distribution of Consumption Shares

1. The random variables $\tau_{1}(t)$ and $\tau_{2}(t)$ have the following density functions on $[0,1]$

$$
\begin{aligned}
& f_{\tau_{1}(t)}(x)=\frac{1}{2 \sqrt{2 \pi t} \eta \delta} \frac{1}{x(1-x)} \exp -\frac{1}{2} \frac{\left[\log \left(\frac{x}{1-x}\right)-\log \left(\frac{\gamma_{1}}{\gamma_{2}}\right)\right]^{2}}{4 \eta^{2} \delta^{2} t} \mathbf{1}_{[0,1]}(x) \\
& f_{\tau_{2}(t)}(x)=\frac{1}{2 \sqrt{2 \pi t} \eta \delta} \frac{1}{x(1-x)} \exp -\frac{1}{2} \frac{\left[\log \left(\frac{x}{1-x}\right)-\log \left(\frac{\gamma_{2}}{\gamma_{1}}\right)\right]^{2}}{4 \eta^{2} \delta^{2} t} \mathbf{1}_{[0,1]}(x)
\end{aligned}
$$

2. For all $t$ and for $i=1,2$, the median of the distribution of the consumption share of each agent is given by her initial "biased wealth level" $\gamma_{i}$, i.e., we have $P\left(\tau_{i}(t)>\gamma_{i}\right)=P\left(\tau_{i}(t)<\gamma_{i}\right)=1 / 2$.

3. As time $t$ increases, $P\left(\tau_{i}(t)\right.$ is near 1$)$ as well as $P\left(\tau_{i}(t)\right.$ is near 0$)$ increase towards $1 / 2$.

C. Consumption Shares Stochastic Processes 
1. The optimal consumption shares are time varying and stochastic. We have $\tau_{i}=\frac{\gamma_{i}\left(M^{i}\right)^{\eta}}{\gamma_{1}\left(M^{1}\right)^{\eta}+\gamma_{2}\left(M^{2}\right)^{\eta}}$. Each agent has a larger share of aggregate endowment in the states that she thinks more probable. The share $\tau_{1}$ is near zero when $e^{*}$ takes very low values, and it is near one when $e^{*}$ takes very high values. The economy is dominated by the optimistic agent for very high levels of aggregate endowment and dominated by the pessimistic agent for very low levels of aggregate endowment. There is a bias towards Agent 1 in the good states of the world and a bias towards Agent 2 in the bad states of the world.

2 . The stochastic processes $\tau_{1}(t)$ and $\tau_{2}(t)$ exhibit mean-reversion. More precisely, they satisfy the following Stochastic Differential Equations

$$
\begin{aligned}
& d \tau_{1}(t)=2 \eta \delta \tau_{1} \tau_{2}\left[\eta \delta\left(\tau_{2}-\tau_{1}\right) d t+d W_{t}\right] \\
& d \tau_{2}(t)=-2 \eta \delta \tau_{1} \tau_{2}\left[\eta \delta\left(\tau_{2}-\tau_{1}\right) d t+d W_{t}\right]
\end{aligned}
$$

\section{Asset Pricing}

1. The instantaneous risk free rate and the market price of risk are time and state varying. They are given by

$$
\begin{aligned}
r_{t}^{f} & =r_{t}^{f}(\text { stdd })+\frac{\sigma}{\eta}\left(\tau_{1}(t)-\tau_{2}(t)\right) \delta-2(\eta-1) \delta^{2} \tau_{1}(t) \tau_{2}(t), \\
M P R_{t} & =M P R_{t}(\text { stdd })-\left(\tau_{1}(t)-\tau_{2}(t)\right) \delta
\end{aligned}
$$

where $r_{t}^{f}($ stdd $)=\frac{\mu}{\eta}-\frac{\sigma^{2}}{2}\left(1+\frac{1}{\eta}\right)$ and $M P R($ stdd $)=\frac{\sigma}{\eta}$. The quantity $\tau_{1}(t) \tau_{2}(t)$ converges to 0 in Probability.

2. The market price of risk is lower in states of the world where $\tau_{1}(t)>\tau_{2}(t)$, i.e. in "good" states of the world and higher in states of the world where $\tau_{2}(t)>\tau_{1}(t)$, i.e. in "bad" states of the world. The risk free rate is higher in "very good" states of the world and lower in "very bad" states of the world.

3. The market price of risk exhibits mean reversion and short run momentum with

$$
M P R_{t}=\frac{\sigma}{\eta}-\delta\left(\tau_{1}(t)-\tau_{2}(t)\right)=\frac{\sigma}{\eta}-\delta\left(2 \tau_{1}(t)-1\right) .
$$

4. The discount rate is always converging to the pessimistic rate in the long run. For $\eta=1 / 2$, we have for all $t$,

$$
R_{t}=2 \mu-3 \sigma^{2}-\frac{1}{t} \log \left[\gamma_{1}^{2} \exp -(2 \delta \sigma t)+\gamma_{2}^{2} \exp (2 \delta \sigma t)+2 \gamma_{1} \gamma_{2} \exp -\frac{1}{2} \delta^{2} t\right]
$$

and $R_{\infty}=2 \mu-3 \sigma^{2}-2 \sigma \delta, \quad R_{0}=2 \mu-3 \sigma^{2}+2 \delta \sigma\left(\gamma_{1}-\gamma_{2}\right)+\frac{1}{4} \delta^{2}\left[1-\left(\gamma_{1}-\gamma_{2}\right)^{2}\right]$. 


\section{E. Consensus Belief}

1. Letting $f_{i}$ denote the density of the distribution of $\log e_{t}^{*}$ for investor $i$, the distribution of $\log e_{t}^{*}$ admits the following densities for the consensus investor $f=\left(\gamma_{1}\left(f_{1}\right)^{\eta}+\gamma_{2}\left(f_{2}\right)^{\eta}\right)^{1 / \eta}$, which is an Hölder average of the initial ones.

2. Since an Hölder average of Gaussian random variables is not Gaussian, $f$ is not Gaussian. Moreover, the distribution of $\log e^{*}$ for the consensus investor is bimodal.

\section{F. Risk Sharing Rule and Trading Volumes}

We have

$$
y^{*^{i}}=\frac{1}{2} e^{*}+\left(\gamma_{i}-\frac{1}{2}\right) e^{*}+\gamma_{i}\left(\frac{\left(M^{i}\right)^{\eta}}{\gamma_{1}\left(M^{1}\right)^{\eta}+\gamma_{2}\left(M^{2}\right)^{\eta}}-1\right) e^{*}
$$

The conclusions of Section 6 remain essentially valid, modulo the bias induced by the $\gamma_{i}$.

\section{G. Volatility}

We consider a stock $\mathrm{S}$, whose dividends are the aggregate endowment process, $d S_{t}+e_{t}^{*} d t=S_{t}\left[\mu_{s} d t+\sigma_{s} d W_{t}\right]$.

We consider the case $\eta=1 / 2$. We suppose that $\rho=0$ and $\mu-\sigma \delta-\sigma^{2}>0$. We have in the standard rational setting $S_{t}=\frac{1}{\mu-\sigma^{2}} e_{t}^{*}$ and $\sigma_{S}=\sigma$. We denote by $S^{\text {opt }}$ (resp. $S^{\text {pess }}$ ) the prices that would prevail if the economy was made of the optimistic (resp. pessimistic) agents only. In the setting with disagreement, we get

$$
\begin{aligned}
S_{t} & =e_{t}^{*}\left[a \tau_{1}^{2}+b \tau_{2}^{2}+2 c \tau_{1} \tau_{2}\right] \\
\sigma_{S} & =\sigma+2 \delta \tau_{2}\left(1-\frac{b \tau_{2}+c \tau_{1}}{a \tau_{1}^{2}+b \tau_{2}^{2}+2 c \tau_{1} \tau_{2}}\right)
\end{aligned}
$$

with $a=\frac{1}{\mu+\delta \sigma-\sigma^{2}}, b=\frac{1}{\mu-\delta \sigma-\sigma^{2}}, c=\frac{1}{\mu+\frac{1}{2} \delta^{2}-\sigma^{2}}$. In particular, in the long run, $\frac{S_{t}}{e_{t}^{*}}$ either converges to $\frac{S_{t}^{1}}{e_{t}^{*}}$ or to $\frac{S_{t}^{2}}{e_{t}^{*}}$ with a probability 1 , and $\sigma_{S}$ converges to $\sigma$.

\section{Proof of G.}

We have

$$
\begin{aligned}
S_{t} & =\frac{\left(e_{t}^{*}\right)^{2}}{M_{t}} E_{t}\left[\int_{t}^{+\infty} M_{s}\left(e_{s}^{*}\right)^{-1} d s\right]=\frac{e_{t}^{*}}{M_{t}} E_{t}\left[\int_{t}^{+\infty}\left(\gamma_{1}^{2} M_{s}^{1}+\gamma_{2}^{2} M_{s}^{2}+2 \gamma_{1} \gamma_{2} \sqrt{M_{s}^{1} M_{s}^{2}}\right)\left(\frac{e_{s}^{*}}{e_{t}^{*}}\right)^{-1} d s\right] \\
& =e_{t}^{*}\left(\gamma_{1}^{2} \frac{M_{t}^{1}}{M_{t}}\right) E_{t}\left[\int_{t}^{+\infty}\left(\frac{M_{s}^{1}}{M_{t}^{1}}\right)\left(\frac{e_{s}^{*}}{e_{t}^{*}}\right)^{-1} d s\right]+e_{t}^{*}\left(\gamma_{2}^{2} \frac{M_{t}^{2}}{M_{t}}\right) E_{t}\left[\int_{t}^{+\infty}\left(\frac{M_{s}^{2}}{M_{t}^{2}}\right)\left(\frac{e_{s}^{*}}{e_{t}^{*}}\right)^{-1} d s\right]+
\end{aligned}
$$




$$
\begin{aligned}
& e_{t}^{*}\left(2 \gamma_{1} \gamma_{2} \sqrt{\frac{M_{t}^{1}}{M_{t}}} \sqrt{\frac{M_{t}^{2}}{M_{t}}}\right) E_{t}\left[\int_{t}^{+\infty}\left(\sqrt{\frac{M_{s}^{1} M_{s}^{2}}{M_{t}^{1} M_{t}^{2}}}\right)\left(\frac{e_{s}^{*}}{e_{t}^{*}}\right)^{-1} d s\right] \\
= & e_{t}^{*}\left[\tau_{1}^{2}(t) a_{t}+\tau_{2}^{2}(t) b_{t}+2 \tau_{1}(t) \tau_{2}(t) c_{t}\right]
\end{aligned}
$$

with $a_{t}=E_{t}\left[\int_{t}^{+\infty}\left(\frac{M_{s}^{1}}{M_{t}^{1}}\right)\left(\frac{e_{s}^{*}}{e_{t}^{*}}\right)^{-1} d s\right], b_{t}=E_{t}\left[\int_{t}^{+\infty}\left(\frac{M_{s}^{2}}{M_{t}^{2}}\right)\left(\frac{e_{s}^{*}}{e_{t}^{*}}\right)^{-1} d s\right]$ and $c_{t}=$ $E_{t}\left[\int_{t}^{+\infty}\left(\sqrt{\frac{M_{s}^{1} M_{s}^{2}}{M_{t}^{1} M_{t}^{2}}}\right)\left(\frac{e_{s}^{*}}{e_{t}^{*}}\right)^{-1} d s\right]$. Elementary algebra yields $a_{t}=\frac{1}{\mu+\delta \sigma-\sigma^{2}}, b_{t}=$ $\frac{1}{\mu-\delta \sigma-\sigma^{2}}, c_{t}=\frac{1}{\mu+\frac{1}{2} \delta^{2}-\sigma^{2}}$.

\section{II- Results for more general models of disagreement}

We have $\tau_{1}(t)=f\left(X_{t}\right)$ with $f(x)=\frac{\exp x}{1+\exp x}$ and $X_{t}=\int_{0}^{t} 2 \delta(s, \omega) W_{s}$. By Itô's Lemma and elementary algebra, we get the following Stochastic Differential Equations

$$
\begin{aligned}
d \tau_{1}(t) & =2 \delta^{2} \tau_{1}\left(1-\tau_{1}\right)\left(1-2 \tau_{1}\right) d t+2 \delta \tau_{1}\left(1-\tau_{1}\right) d W_{t} \\
& =2 \delta \tau_{1} \tau_{2}\left[\delta\left(\tau_{2}-\tau_{1}\right) d t+d W_{t}\right] \\
d \tau_{2}(t) & =-2 \delta \tau_{1} \tau_{2}\left[\delta\left(\tau_{2}-\tau_{1}\right) d t+d W_{t}\right]
\end{aligned}
$$




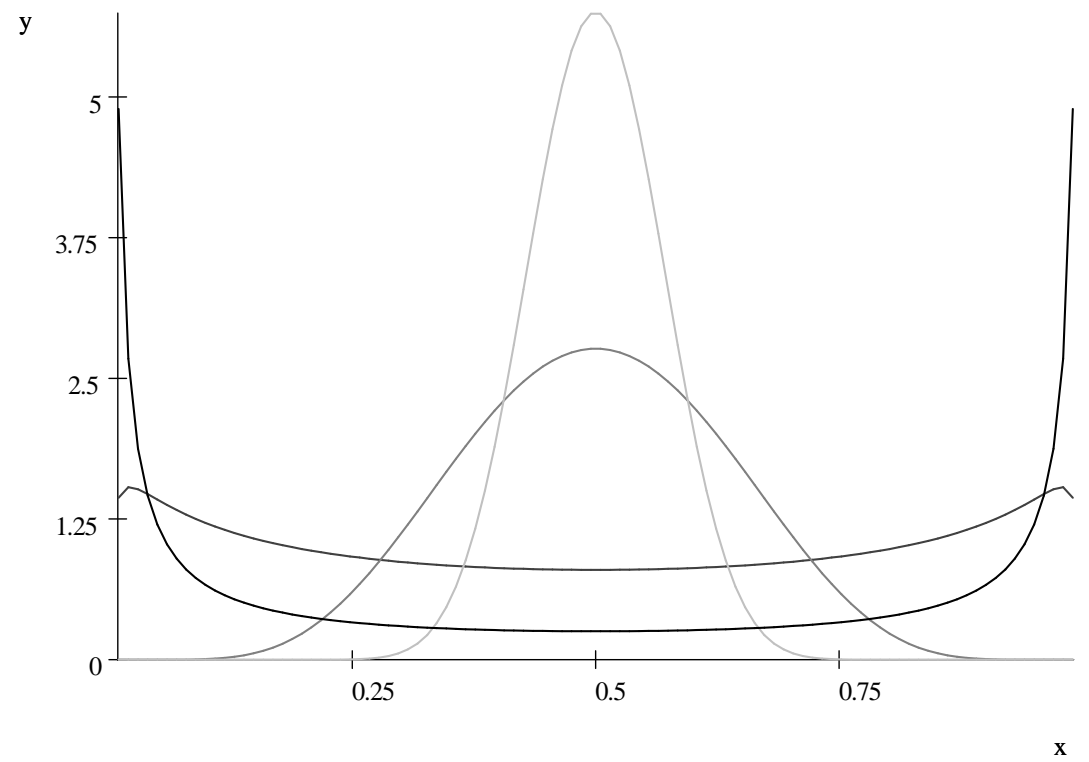

Figure 1: This Figure represents the distribution of $\tau_{1}(t)$ (or equivalently of $\left.\tau_{2}(t)\right)$ for different horizons $t=$ week, 1 month, 1 year and 10 years. We take $\delta=$ 1 which means that agent 1 (resp. agent 2) overstimates (resp. underestimates) the drift by one times the volatility. The curve with the highest peak corresponds to a one week horizon: both agents have their weight concentrated around 0.5. The curve has the same shape but is more spread out for the one month horizon. It becomes more and more concave with all the mass concentrated around 0 and 1 when the horizon increases to 1 year and then to 10 years. The shape of the distribution of the market price of risk is homothetic to the shape of the distribution of $\tau_{1}(t)$. It suffices to replace the $[0,1]$ support by $[\sigma(1-\delta), \sigma(1+\delta)]$. 


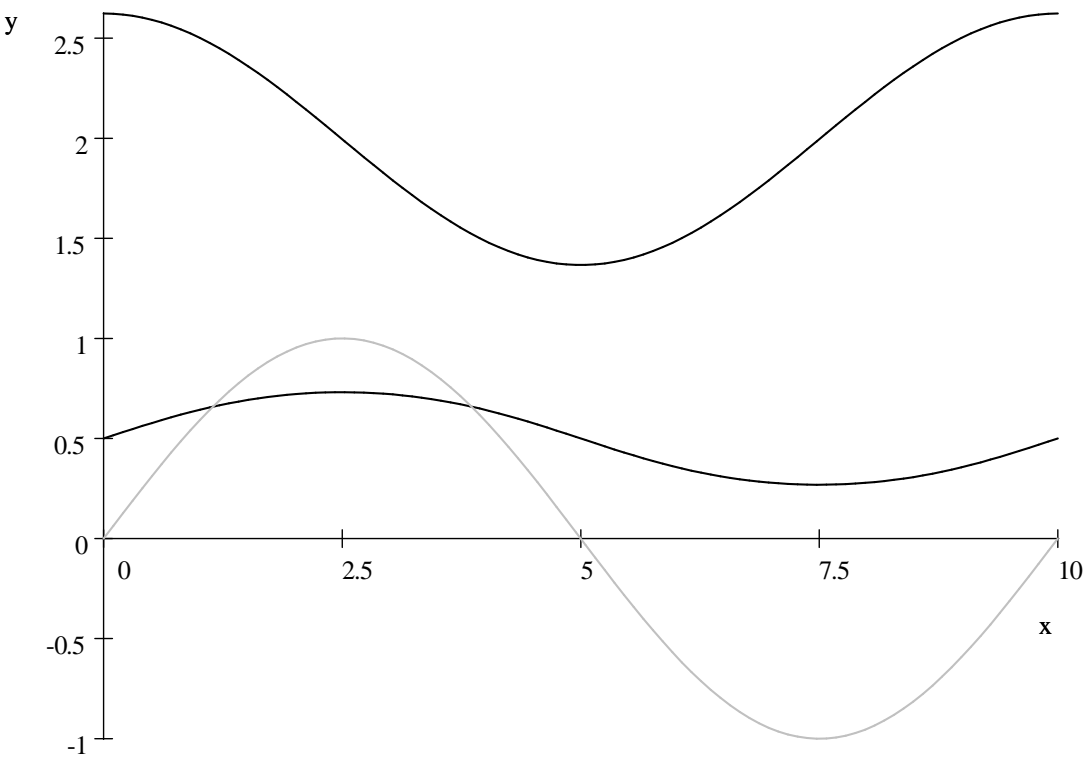

Figure 2: In this figure, the grey curve represents a (smoothed) sample path of the Brownian motion that describes a business cycle over a period of 10 years. The intermediate curve represents the evolution of the optimistic agent consumption share and the higher curve represnts (in \%) the associated instantaneous growth rate of the economy. The associated evolution of the market price of risk and of the risk free rate are represented in Figure 3. 


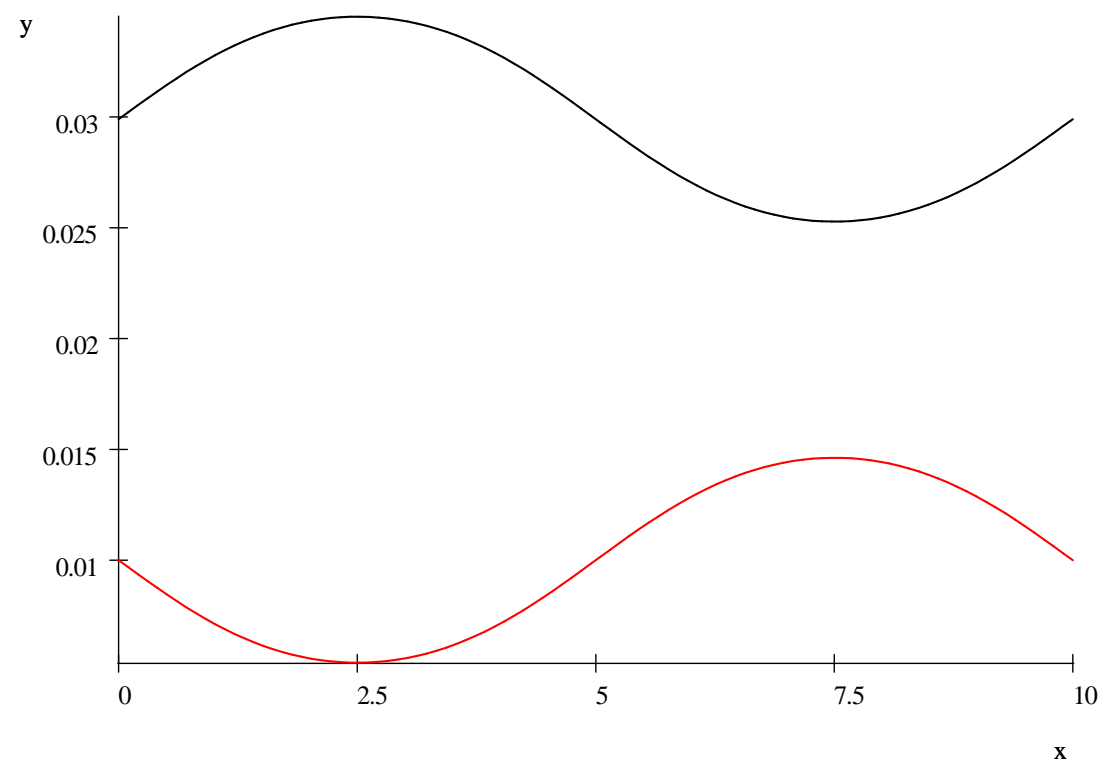

Figure 3: In this figure, the higher curve represents the evolution of the short term rate that is associated to the business cycle described in Figure 2 and the lower curve represents the evolution of the market price of risk. 